\title{
An annotated checklist of myxomycetes from the Seychelles Islands, Indian Ocean
}

\section{Tetiana Kryvomaz ${ }^{*}$, Alain Michaud ${ }^{2}$ \& Steven L. Stephenson ${ }^{3}$}

1 Kyiv National Construction and Architecture University, Povitroflotskyi prospect 31, Kiev UA-03680, Ukraine

293 route de la Croizette, FR-38360, Engins, France

3 University of Arkansas, Department of Biological Sciences, Fayetteville, USA-72701 Arkansas, USA

* Corresponding author: ecol@i.ua

Keywords: Amoebozoa, biodiversity, island myxomycetes, plant substrates, species inventory, tropics.

Article info:

Article info:

Received: 09 August 2020

Accepted: 23 September 2020

Published online: 23 October 2020

Corresponding Editor: Nikki Heherson A. Dagamac

\section{Abstract}

The checklist provided herein contains 143 species and infra-specific taxa of myxomycetes representing six orders, 12 families and 29 genera known from the Seychelles Islands. These records are the result of 878 field collections and 468 samples processed with the use of the moist chamber techinque. The overall study involved expeditions to the granitic group of islands Mahé, Praslin, La Digue, Curieuse, Félicité, and data from the literature for the coral Aldabra atoll. The taxonomic structure of the myxomycete biota for the islands studied indicates a predominance of members of the order Physarales (74 taxa). The main genera are Physarum (38 species and two varieties), Didymium (17 species), Cribraria (11 species), Arcyria (eight species) and Stemonitis (six species and two varieties). For all six islands only a single species of myxomycete (Physarum crateriforme) was shared in common. For the total assemblage of species recorded from all of the islands, $4 \%$ species were abundant, $12 \%$ species were common, $29 \%$ were found occasionally, $42 \%$ were rare, and $13 \%$ species had only a single record. The most abundant species were Arcyria cinerea, A. de- 
nudata, Diderma effusum, Hemitrichia calyculata, Physarum compressum, and P. melleum. Based on data from 50 different localities with 90 collecting plots, $32 \%$ of all specimens were associated with coastal vegetation, $30 \%$ with lowland localities, $19 \%$ with intermediate forests, $9 \%$ with riverine forests, $8 \%$ with mountain forests, and only $2 \%$ with mangrove swamps. In general, this annotated checklist clearly shows that isolated tropical islands can support a diverse assemblage of myxomycetes.

\section{Introduction}

Although a few studies of the myxomycetes of particular islands have been carried out by authors (e.g., Adamonyte et al. 2011, Macabago et al. 2012, 2016, 2017, Kryvomaz et al. 2017, 2020, Stephenson \& Stephenson 2019, 2020), there is still not enough information available on myxomycetes to address some of the fundamental questions relating to such things as long-distance dispersal and gene flow as they relate to these organisms. The primary purpose of the expeditions described in this paper was to contribute to the body of data available on the island biogeography of myxomycetes. The annotated checklist provided herein contains the result of expeditions to the granitic group of islands in the Seychelles during the period of 2011-2016. There were three expeditions to La Digue, two expeditions to the Mahé, and one expedition each to Praslin, Curieuse and Félicité. In addition to the records obtained a result of these expeditions, myxomycete reported in the literature from the coralline Aldabra atoll were included in the checklist (Ing \& Hnatiuk 1981).

\section{Materials and methods}

the Seychelles archipelago consists of 115 granitic and coral islands near the equator in the Western Indian Ocean between Africa and Asia (Fig. 1). The largest island is Mahé $\left(142 \mathrm{~km}^{2}\right)$, which reaches an elevation of $905 \mathrm{~m}$ at Morne Seychellois, where expeditions took place in October 2011 and in June 2016. Myxomycetes were studied on Praslin (elevation $367 \mathrm{~m}, 38 \mathrm{~km}^{2}$ ) in June-July 2015. There were three expeditions to La Digue (elevation $333 \mathrm{~m}, 10$ $\mathrm{km}^{2}$ ), which took place in July 2015, in January 2016 and July 2016. Some additional material was collected on two small islands with limited access. These were Curieuse $\left(2.9 \mathrm{~km}^{2}\right)$ in June 2015 and Félicité $\left(2.68 \mathrm{~km}^{2}\right)$ in July 2016 .

The climate of the Seychelles is a warm and rather constant humid tropical type with a strong maritime influence (Stoddart 1984). The year can be divided into two main seasons - the rainy season that runs from October to April and the Southeast Monsoon which extends from May to October. The driest period lasts from June to August (Hansen \& Laboudallon 2013).

The native flora of the Seychelles includes elements of African, Madagascan and Indo-Malaysian origin, $34 \%$ of which are thought to be endemic to the Seychelles (Fleischmann et al. 2003). The primary natural vegetation types of the Seychelles are the coastal plateau, coastal and lowland forests, mangrove forests, riverine forests, intermediate forests, mountain mist forests and the glacis type vegetation (Merlin et al. 2012). The plots where collecting was carried out during expeditions were selected with a consideration these basic types of vegetation.

In the coastal vegetation (CV) type, most plants of the plants present along the coast are species common to the shores of most tropical islands. The coastal forest is found along the edges of sandy beaches and also on the granite coasts. The dominant species are Calophyllum inophyllum L., Scaevola taccada (Gaertn.) Roxb., Cordia subcordata Lam., Hibiscus tiliaceus L. and Cocos nucifera L. (Fleischmann et al. 2003). The indigenous takamaka (Calophyllum inophyllum) can be found up to $300 \mathrm{~m}$ above sea. Numerous lianas grow near the coasts, with such species as Ipomoea carica Sweet and I. macrantha Roem. \& Schult. (Hansen \& Laboudallon 2013). Also near sea level are mangrove swamps (MS) dominated by Avicennia marina (Forssk.) Vierh. and Rhizophora mucronata Lam. (Fleischmann et al. 2003).

The lowland forests (LF) originally covered the mountain slopes up to about 200-300 m above sea 


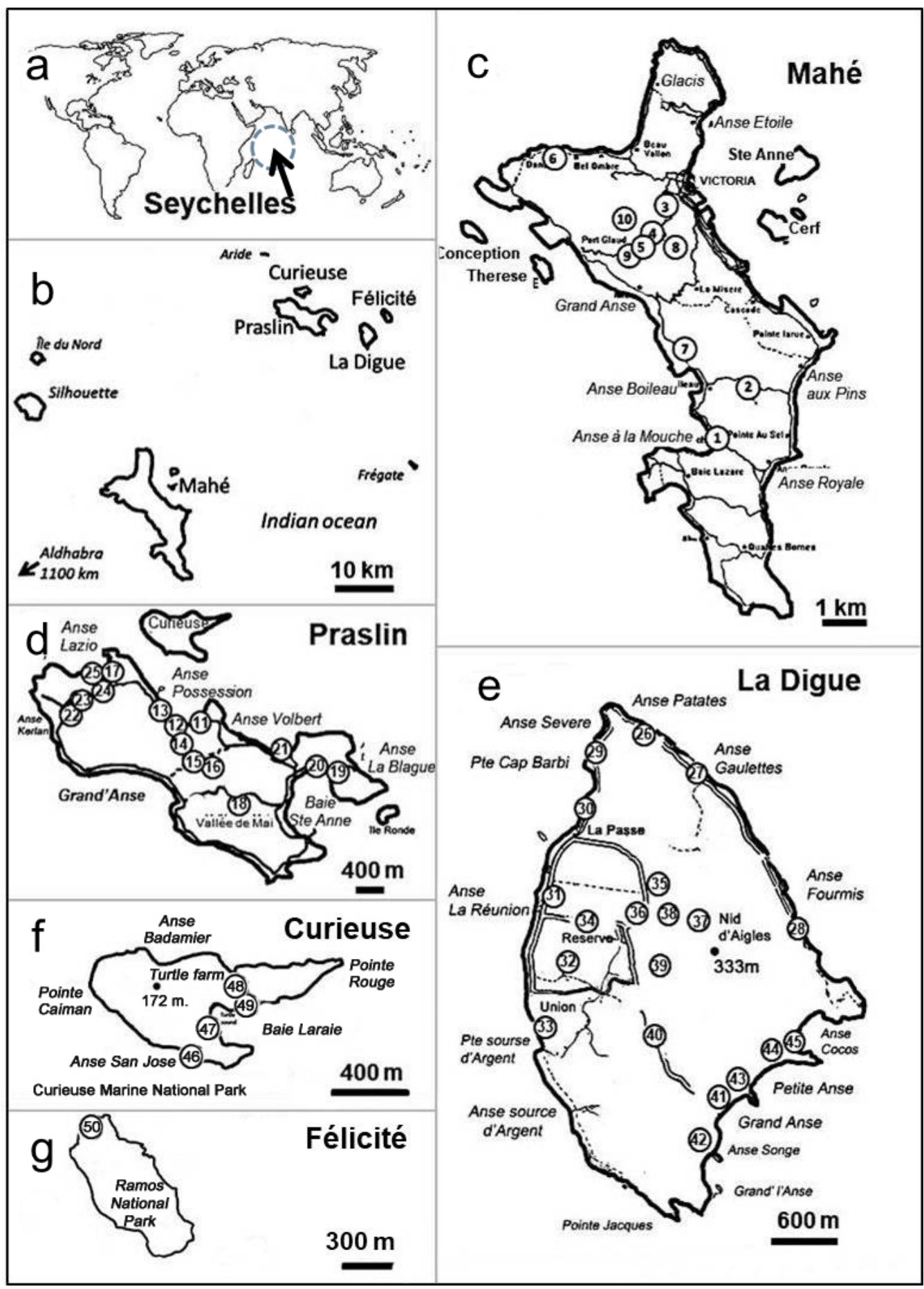

Fig. 1. Map of the Seychelles with collecting localities indicated by numbers and circles (details are provided in the Materials and Methods section). a: The Seychelles on a world map, b: The Seychelles inner islands (the granitic group), c: Mahé, d: Praslin, e: LaDigue, f: Curieuse, g: Félicité. 
level, but in the present study low elevation localities were considered to be those no more than about 90 $m$ above sea level (Hill 2002). The species of plants present are not dispersed by ocean currents as is the case for many coastal species. The primary lowland flora was apparently composed partly of endemic species as well as indigenous species more widespread on islands in the Indian Ocean (Fleischmann et al. 2003). In drier and less accessible localities, the endemic Deckenia nobilis H.Wendl. ex Seem. is often a dominant species (Hansen \& Laboudallon 2013). Most of the remaining riverine forests (RF) in the Seychelles are composed of palm trees and vegetation along rivers that has been greatly affected by human activities. There also seems to be a constant association of Martellidendron hornei (Balf.f.) Callm. \& Chassot (Fleischmann et al. 2003). Lowland and coastal localities examined included beach vegetation, beach fringes along roadsides, muddy coastal areas with mangroves present, coastal sunny exposed scrublands, rocky or sandy woodlands, wet lowland shady forests, woodlands in wet river beds, semi-humid slope forests, vegetation near habitations, and recreation areas, public parks and gardens, and agricultural land.

The intermediate forest (IF) zone of the Seychelles extends from 200 to $500 \mathrm{~m}$, but in this study it was considered to range from 90 to $300 \mathrm{~m}$ above sea level. These forests are rich in species and have a high canopy, occasionally reaching up to 30-40 m (Franda 2019). On drier sites the intermediate forests are largely dominated by the endemic palm trees. Palms were of only minor importance in the forests of the more humid type (Merlin et al. 2012). The studied mid-elevations localities included humid forests with a considerable amount of plant remains in moist forests, on dryer sunny exposed rocky areas, open scrublands, sunny exposed woodland, slopes with red soil in association with bracken ferns, public parks or plant remains in reserves, semi-humid woodlands near habitations, and agricultural land or old plantations.

The mountain forests (MF) originally covered most of the land above 400-500 $\mathrm{m}$ in the Seychelles. The highest peak in the Seychelles is Morne Seychellois $(914 \mathrm{~m}$ ) in the central portion of Mahé (Hansen \& Laboudallon 2013). This elevation range included high elevation rain forests, hygrophile and moist for- ests. The vulnerable endemic Northea hornei Pierre is typical of this type of forest (Merlin et al. 2012). The mountain mist forest is rich in mosses, lichens, filmy ferns and epiphytic orchids. Large trees can still be found at undisturbed sites at higher elevations, indicating that the canopy previously reached a height of at least $15 \mathrm{~m}$. In the original forests at higher elevations, endemic species dominated the vegetation (Fleischmann et al. 2003).

In total, 50 different localities with 90 collecting plots were subjected to some sampling. At each locality, the microhabitats in which myxomycetes are known or suspected to occur were examined systematically. Each plot was representative of a particular type of forest community of the main vegetation types of the Seychelles with respect to both vegetation and site conditions and consisted of a relatively homogeneous unit of vegetation located in an area of essentially uniform topography. Sampling was repeated to obtain a series of substrate samples of each type following the procedure described by Stephenson \& Rojas (2017).

\section{List of localities:}

Mahé: LOC. 1: 13-X-2011, Glacis district, Anse Nord d'Est, CV, alt. 3 m, -4.56995, 55.45791. LOC. 2: 27-VI2016, Bel Ombre district, Anse Major trail, LF, alt. 55 m, -4.61883, 55.39717. Loc. 3: 14-X-2011, Port Glaud district, Anse Souillac, Cap Ternay road, CV, alt. $8 \mathrm{~m}$, -4.64706, 55.39124. Loc. 4: 14-X-2011, Port Glaud district, Port Launay, Morne Seychellois National Park, LF, alt. 20 m, -4.65145, 55.40004. LOC. 5: 10-X2011, Port Glaud, Port Launay road, Port Glaud district, CV, alt. 3 m, -4.66255, 55.41265. LOC. 6: Morne Seychellois mountain, Sans Soucis road: A: 26-VI2016, Mont Fleuri district, IF, alt. 223 m,-4.63482, 55.45009; B: 30-VI-2016, Port Glaud district, Tea factory, MF, alt. $399 \mathrm{~m},-4.66247,55.43767$; C: 09-X-2011, MF, alt. 420 m, -4.65595, 55.44597; D: 29-VI-2016, Bel Air district, Salazie trail, MF, alt. 430 m, -4.65283 , 55.44862; E: 26-VI-2016, Port Glaud district, MF, alt. 448 m, -4.65401, 55.44518; F: 26-VI-2016, Port Glaud district, MF, alt. 458 m,-4.65298, 55.44498; G : 30-VI2016, Port Glaud district, Morne Blanc trail, MF, alt. 
458 m, -4.65937, 55.43747. LOC. 7: 11-X-2011, Boileau district, Anse Anse Boileau, West coast road, LF, alt. 40 m, -4.703950, 55.476310. LOC. 8: 28-VI-2016, Grand' Anse district, National Biodiversity Centre Barbarons, LF, alt. 76 m, -4.69281, 55.46512. LOC. 9: 25-VI-2016, La Reserve trail, IF, alt. 245 m, -4.70694, 55.5005. LOC. 10: Baie Lazare district, Anse à la Mouche: A: 11-X-2011, West coast road, CV, alt. 5 m, -4.740675, 55.480450; B: 24-VI-2016, CV, alt. 4 m, -4.74015, 55.4892. LOC. 11: 11-X-2011, Baie Lazare district, Bay Lazare, West coast road, CV, alt. $8 \mathrm{~m}$, -4.765641, 55.484411. LOC. 12: 11-X-2011, Takamaka district, Anse Takamaka, West coast road, CV, alt. 10 m, -4.77249, 55.49125. LOC. 13: 12-X-2011, Takamaka district, Le Reduit, Mont Fleuri road, LF, alt. 60 m, -4.77666,55.50108. LOC. 14: 12-X-2011, Takamaka district, Anse Intendance, CV, alt. 15 m, -4.78290, 55.50219. LOC. 15: 11-X-2011, Takamaka district, Anse Petite Police, Grand Police Bay road, CV, alt. 7 m, -4.80228, 55.51673. LOC. 16: 12-X-2011, Takamaka district, Police Bay, Chemin Grande Police, RF, alt. 5 m, -4.80295, 55.52077. LOC. 17: 12-X-2011, Takamaka district, Anse Parnel, South coast road, CV, alt. 6 m, -4.77021, 55.52348. LOC. 18: 10-X-2011, Anse Royale district, Anse Royale, South coast road, CV, alt. $7 \mathrm{~m},-4.74258,55.51666$.

PRASLIN: LOC. 19: 2-VII-2015, Anse Lazio, CV, alt. 5 m, -4.2941, 55.70274. LOC. 20: 7-VII-2015, Anse Lazio to Anse Lemuria, Savoy state Land, LF, alt. 40 m, -4.30288, 55.69204. LOC. 21: Anse Kerlan: A: 8-VII2015, Anse Kerlan Estate, LF, alt. 39 m, -4.29629, 55.6962; B: 8-VII-2015, Anse Kerlan Estate, LF, alt. 48 m, -4.29619, 55.6948; C: 5-VII-2015, Anse Kerlan to Savoy State Land, LF, alt. 47 m, -4.30414, 55.6867; D: 5-VII-2015, Anse Kerlan to Savoy State Land, IF, alt. 99 m, -4.30555, 55.6889; E: 5-VII-2015, Anse Kerlan to Savoy State Land, IF, alt. 132 m, -4.30083, 55.69246; F: 5-VII-2015, Newcome to Savoy State Land, IF, alt. 104 m, -4.30398, 55.6899. LOC. 22: Possession: A: 28-VI-2015, Anse Takamaka, CV, alt. 12 m, -4.30761, 55.71896; B: 28-VI-2015, Anse Pasquière, CV, alt. 5 m, -4.31149, 55.72122; C: 27-VI-2015, Possession Estate, LF, alt. 50 m, -4.31428, 55.7301; D: 30-VI-2015, Anse Possession to Grand Anse, Pasquière Estate, LF, alt. 72 m, -4.32643, 55.72341; E: 30-VI-2015, Anse Possession to Grand' Anse, Pasquière Estate, IF, alt. 101 m, -4.31907, 55.7234; F: 30-VI-2015, Anse Possession to Grand' Anse, Salazie, IF, alt. 117 m, -4.3247,
55.72396; G: 30-VI-2015, Anse Possession to Grand' Anse, La Plaine hollandaise, IF, alt. 119 m, -4.32401, 55.72429. LOC. 23: 3-VII-2015, Vallée de Mai, IF, alt. 179 m, -4.33185, 55.7401. LOC. 24: 4-VII-2015, Anse Gouvernement, CV, alt. 7 m, -4.32104, 55.76104. LOC. 25: Anse Takamaka: A: 3-VII-2015, Baie Ste. Anne, MS, alt. 12 m, -4.32653, 55.77244; B: 3-VII-2015, Anse Takamaka to Anse La Blague, CV, alt. 1 m, -4.32654, 55.76661; C: 3-VII-2015, Anse Takamaka to Anse La Blague, CV, alt. $1 \mathrm{~m},-4.32654,55.76661$.

LA DIGUE: LOC. 26: 4-VII-2016, Anse Patates, CV, alt. 11 m, $-4.33769,55.83306$. LOC. 27: Anse Gaulettes: A: 5-I-2016, RF, alt. 9 m, -4.33988, 55.83652; B: 4-VII-2016, CV, alt. 4 m,-4.340350, 55.83734; C: 4-VII-2016, RF, alt. 9 m, $-4.34077,55.8372$. LOC. 28: Anse Fourmis: A: 9-I-2016, CV, alt. 22 m, -4.35633, 55.8493; B: 9-I-2016, CV, alt. $31 \mathrm{~m},-4.35888,55.85055$. LOC. 29: 4-VII-2016, CV, alt. 3 m, $-4.34184,55.8304$. LOC. 30: 4-I-2016, Pointe Cap Barbi, LF, alt. 16 m, -4.34396, 55.83094. LOC. 31: Anse La Réunion: A: 2-I-2016, LF, alt. 7 m, -4.35357, 55.82742; B: 3-I-2016, LF, alt. 16 m, -4.35692, 55.83019; C: 6-VII-2015, LF, alt. $16 \mathrm{~m},-4.35773,55.8303$. LOC. 32: 8-I-2016, Union to Grand Anse, LF, alt. 5 m, $-4.36224,55.82804$. LOC. 33: 9-I-2016, Union, LF, alt. 8 m, -4.36597, 55.8269. LOC. 34: La Passe: A: 2-I-2016, LF, alt. 9 m, -4.34962, 55.8304; B: 6-I-2016, IF, alt. 126 m, -4.35013, 55.83734; C: 13-I-2016, LF, alt. $27 \mathrm{~m},-4.35289$, 55.83607. LOC. 35: 05-I-2016, La Passe, LF, alt. $30 \mathrm{~m}$, -4.35498, 55.8360. LOC. 36: 8-VII-2016, near Natural Reserve La Veuve, LF, alt. 9 m, -4.35655, 55.8340. LOC. 37: The road to Belle Vue, Nid d'Aigles: A: 5-VII2016, LF, alt. $80 \mathrm{~m},-4.35528,55.84305$; B: 5-VII-2015, IF, alt. 275 m, -4.35759, 55.8427; C: 5-VII-2015, IF, alt. 277 m, -4.35756, 55.84275; D: 5-VII-2015, IF, alt. 293 m, -4.35684, 55.84345; E: 13-I-2016, IF, alt. $133 \mathrm{~m}$, $-4.35786,55.83982$; F: 3-I-2016, IF, alt. 163 m, -4.35859, 55.84031; G: 3-I-2016, IF, alt. $160 \mathrm{~m},-4.35878$, 55.84023; H: 3-I-2016, IF, alt. 147 m, -4.35861, 55.8399; I: 13-I-2016, IF, alt. $126 \mathrm{~m},-4.35854,55.83963$; J: 5-VII-2016, IF, alt. $160 \mathrm{~m},-4.35893,55.84018$; K: 5-VII-2016, IF, alt. $182 \mathrm{~m},-4.35904,55.84059$; L: 5-VII2016, IF, alt. $212 \mathrm{~m},-4.35775,55.84138$; M: 3-VII2016, LF alt. 60 m, -4.35806, 55.83719; N: 13-I-2016, IF, alt. $111 \mathrm{~m},-4.36009,55.83867$; 0: 3-I-2016, IF, alt. $106 \mathrm{~m},-4.35993,55.83845$; P: 3-I-2016, IF alt. 128 m, -4.36029, 55.83920. LOC. 38: Réunion to Grand Anse: A: 7-I-2016, LF, alt. 21 m, -4.36016, 55.83470; 
B: 6-VII-2015, LF, alt. 15 m, -4.36043, 55.8344. LOC. 39: Union - Maurice Payet river: A: 7-VII-2016, RF, alt. $40 \mathrm{~m},-4.36285$, 55.83712; B: 12-I-2016, LF alt. $21 \mathrm{~m},-4.36341,55.83583$; C: 12-I-2016, LF, alt. $16 \mathrm{~m}$, -4.36374, 55.8355. LOC. 40: Union to Grand Anse: A: 8-I-2016, RF, alt. $19 \mathrm{~m},-4.36454,55.83498$; B: 8-I2016, LF, alt. $27 \mathrm{~m},-4.36016$, 55.8347; C: 8-I-2016, LF, alt. 42 m, -4.36689, 55.83645; D: 10-I-2016, LF, alt. $55 \mathrm{~m},-4.36915,55.83759$. LOC. 41: Grand Anse: A: 6-VII-2015, CV, alt. $9 \mathrm{~m},-4.37305,55.8419$; B: 11I-2016, CV, alt. 6 m, -4.37345, 55.84314. LOC. 42: Grand Anse to Anse Songe: A: 3-VII-2016, CV, alt. $21 \mathrm{~m},-4.37503,55.84158$; B: 10-I-2016, CV, alt. 14 m, -4.37628, 55.84206; C: 11-I-2016, RF, alt. $12 \mathrm{~m}$, -4.37609, 55.84225. LOC. 43: 10-I-2016, Grand Anse to Petite Anse, CV, alt. 14 m,-4.37196, 55.84513. LOC. 44: 2-VII-2016, Petite Anse, CV, alt. 7 m, -4.36996, 55.8462. LOC. 45: Petite Anse to Anse Cocos: A: 2-VII-2016, CV, alt. $17 \mathrm{~m},-4.36889,55.84638$; B: 2-VII2016, CV, alt. 30 m, -4.36903, 55.84845; C: 2-VII-2016, CV, alt. 41 m, -4.36842, 55.8487; D: 2-VII-2016, CV, alt. $16 \mathrm{~m},-4.36828,55.8499$.

CURIEUSE: LOC. 46: 29-VI-2015, Anse Saint José, CV, 14 m, -4.29019, 55.72624. LOC. 47: Anse Saint José to giant turtles farm: A: 29-VI-2015, CV, 19 m, -4.28748, 55.72838; B: 29-VI-2015, MS, 19 m, -4.28748, 55.72838. LOC. 48: 29-VI-2015, Baie Laraie, giant turtles farm, RF, $13 \mathrm{~m},-4.28254,55.73157$. LOC. 49: 29-VI-2015, RF, 11 m, -4.28303, 55.73126.

FÉLICITÉ: LOC. 50: 6-VII-2016, CV, $4 \mathrm{~m}$, $-4.31736,55.86806$.

The numbers of collections sites on coralline Aldabra atoll are presented here as in Ing \& Hnatiuk (1981): 1 - Ile Picard, 2 - Anse Var, 3 - Point Hodoul, 4 - Cinq Cases, 5 - Ile Malabar, 6 - Islet at Entre Deux, 7 - Anse Tamarind, 8 - Anse Mais, 9 - Ile Esprit, 10 - Islets in the south-west corner of the lagoon at south of Ile Esprit, 11 - Polymnie, 12 - Islets in the lagoon south of Polymnie, 13 - Islets in the lagoon south-east of Passe Houareau, 14 Dune Jean Louis, 15 - Anse Anglais, 16 - near Point Grande Terre.

During the part of this study directed towards the granitic islands, 57 of the species of plants found in the Seychelles were recorded as myxomycete substrates in field collections and/or in moist chamber cultures. The identification of the vegetation was carried out using the book "Flora of the Seychelles" by Hansen \& Laboudallon (2013), with some references to the website http://www.seychellesplantgallery.com. Authors of scientific names of plants were provided when a species was mentioned for the first time.

Field collecting (FC) of specimens of myxomycetes and samples for preparation of moist chamber (MC) cultures were carried out at each locality during the rainy and dry seasons by Kryvomaz and Michaud. MC cultures were prepared with samples of various substrates such as the bark of living trees, aerial litter and ground litter by Stephenson and Michaud. Samples consisting of substrate material were placed on filter paper in Petri dishes. Distilled water adjusted to $\mathrm{pH} 7.0$ was added to each Petri dish, and what had become a moist chamber culture was maintained under diffuse daylight and at room temperature $\left(22-23{ }^{\circ} \mathrm{C}\right)$. Water was added every few days as required to maintain moist conditions for the entire observation period of up to three months. The $\mathrm{pH}$ of each moist chamber culture (taken at 24 hours) was recorded in all instances by Stephenson. Samples were examined with the use of a high-magnification Zeiss Axiostar dissecting microscope every day for the first two weeks and subsequently every 2-3 days for the entire period of observation. Mature fruiting bodies were removed when present and placed in small pasteboard boxes for permanent storage. A species recorded from one moist chamber culture was regarded as a single specimen, irrespective of the number of fruiting bodies appearing or the number of days separating their appearance. Myxomycete abundance was classified according to the ACOR scale based upon the proportion of a species to the total number of records for each survey: A - abundant (>3\%), C common (> 1.5-3\%), $\mathrm{O}-$ occasional $(\geq 0.5-1.5 \%), \mathrm{R}$ - rare $(<0.5 \%)$ (Stephenson et al. 1993).

Determinations of specimens were made with the use of "Les Myxomycètes" (Poulain et al. 2011). Nomenclatural treatment follows Nomenmyx (Lado 2005-2020) with the exception of infra-specific taxa of myxomycetes, as noted in "Les Myxomycètes" (Poulain et al. 2011). All microscopic measurements and observations were made with material mounted in water and a solution of sodium hexametaphosphate. All photographs reproduced in this paper were taken by Michaud. The light photomicrographs were 
obtained using an Olympus TG3 on a Carl Zeiss Axiostar microscope with the Zeiss Acroplan 40x, 100x, 400x, 630x, and 1000x lenses. Macrophotographs of myxomycetes were taken with an Olympus TG3 and a Nikon D70 camera mounted on a Nikon PB6 bellows, with an AF Micro Nikkor $60 \mathrm{~mm}$ or Nikon AF Nikkor $28 \mathrm{~mm}$ lens used in conjunction with an inversion ring along with a Nikon SB-26 flash for supplemental lighting. Photographs of plants were taken in situ with a Nikon D5300 with a lens Nikon AF-S Nikkor 28-300mm 1:3.5-5.6 G-ED VR, a DMC Panasonic TZ20, and an Olympus TG3. The permanent collection of specimens collected in the Seychelles is preserved in the publicly available herbarium of the University of Arkansas (UARK)s, and the private herbaria of Michaud in France and Kryvomaz in the Ukraine.

\section{Results}

A total of 143 species and infra-specific taxa of myxomycetes from the Seychelles are listed in the annotated checklist provided herein. These records are based on 878 field collections and 468 samples processed using the moist chamber method as result of expeditions to granitic group of islands Mahé, Praslin, La Digue, Curieuse, Félicité. The checklist also includes the data from the literature for the coralline Aldabra atoll (Ing \& Hnatiuk 1981). The specimens are listed first by genus and then by species in alphabetical order. Details of collecting localities and data are given in the Material and Methods section, so only the numbers of the locality are provided. In order to save space, the types of information associated with each specimen collected are abbreviated as follows: FC - field collection, $\mathrm{MC}$ - moist chamber (species obtained in moist chamber cultures in the laboratory), $\mathrm{cp}$ - collecting plots, ps plant substrate species, A - abundant, $\mathrm{C}$ - common, $\mathrm{O}$ - occasional, $\mathrm{R}$ - rare, $\mathrm{S}$ - singleton record, Loc - locality. Names of plant substrates are given with authors only for the first mention of the plant in questions. The type of substrates is abbreviated as follows: ar - aerial litter, which includes dead plant parts still attached to living trees, and also epiphytic or parasitic plants on trees; $b$ - bark of trees or stem surface of palms; gl - ground litter, plant remains; w - wood (trunks, stumps, big brunches) and big stem of palms; ; - living plant, ${ }^{+}$- dead or decaying substrates. Specimens cited herein are deposited in the herbarium of the University of Arkansas, Fayetteville, USA under the acronym SLS, which refers to Stephenson, as well as in the private collections of Michaud (AM) and Kryvomaz (TK).

Arcyria cinerea (Bull.) Pers. (Granitic islands: 95 FC, $62 \mathrm{MC}, 50 \mathrm{cp}, 30 \mathrm{ps}$. A)

Aldabra atoll 1, 8, 9, 10, 12: gl+ Cocos sp., $\mathrm{w}^{+}$, 10 gatherings (Ing \& Hnatiuk 1981). LOC. 1: b*, AM2167, TK81S. LOC. 2: $\mathrm{ar}^{+}$Agave angustifolia Haw., SLS32320 (pH 8.1), SLS32321 (pH 7.2). gl ${ }^{+}$Epipremnum pinnatum (L.) Engl., SLS32326 (pH 7.1). $\mathrm{gl}^{+}$Cheilocostus speciosus (J.Konig) C. Specht, SLS32410 (pH 7). LOC. 3: $\mathrm{gl}^{+}$Agave, AM2183, TK97S. b*, SLS26419 (pH 8.6), SLS26721 ( $\mathrm{pH} 7.7)$, SLS26154 ( $\mathrm{pH} 7.9)$, SLS26155 (pH 7.9), SLS26736 (pH 7.4), SLS26744 (pH 8.5), SLS26750 (pH 8.4), SLS26758 (pH 7.9), SLS26764 (pH 8.2), SLS26878 (pH 7.6), SLS26160 (pH 8.5). LOC. 5: $\mathrm{w}^{+}$Plumeria alba L., AM2185, TK99S. LOC. 6C: $\mathrm{W}^{+}$, AM2088, TK2S. LOC. 6D: ar $^{+}$Dianella ensifolia (L.) DC. gl ${ }^{+}$Ipomoea cairica, SLS26770 (pH 6.9), SLS26780 (pH 6.7), SLS26790 (pH 6.1). LOC. 6E Mahé: ar* Nephrolepis biserrata (Sw.) Schott on Elaeis guineensis Jacq., SLS26750 (pH 6.4). b* Tabebuia pallida (Lindl.) Miers, SLS29994 (pH 6.8). ar $^{+}$Thunbergia grandiflora (Roxb. ex Rottler) Roxb., SLS26740 (pH 6.4). LOC. 6G: b* Deckenia nobilis, AM3123, TK2969. $\mathrm{gl}^{+}$Pandanus balfourii Martelli, AM3133, TK2984. $\mathrm{w}^{+}$Tabebuia pallida, AM3151, TK3020. $\mathrm{w}^{+}$AM3141, TK2996. LOC. 8: $\mathrm{w}^{+}$Spondias cytherea Sonner., AM3102, TK2917. gl ${ }^{+}$, AM3111, TK2930. LOC. 9: $\mathrm{w}^{+}$ \& $\mathrm{b}^{+}$Swietenia macrophylla King, AM3043, TK2792. $\mathrm{w}^{+}$Swietenia macrophylla, AM3055, TK2807. $\mathrm{gl}^{+}$ Cheilocostus speciosus, SLS32410 ( $\mathrm{pH}$ 7). LOC. 12: $\mathrm{b}^{*}$ Cocos nucifera, AM2125, TK39S. ar ${ }^{+}$Cocos nucifera. $\mathrm{w}^{+}$with Physarum lakhanpalii, AM2123, TK37S. LOC. 15: $\mathrm{w}^{+}$, AM2129, TK43S. LOC. 19: $\mathrm{ar}^{+}$Musa sp. SLS23874 (pH 6.9). LOC. 20: ar $^{+}$Syngonium podophyllum, SLS26710 (pH 7.7), SLS26720 (pH 7.8). $\mathrm{w}^{+}$ Calophyllum inophyllum AM2783, TK2202. LOC. 21B: $\mathrm{w}^{+}$Terminalia catappa L, AM2789, TK2210. LOC. 21E: $\mathrm{w}^{+}$Phoenicophorium borsigianum (K. Koch) Stuntz., AM2778, AM2807, TK2194. LOC. 21F: $\mathrm{w}^{+}$Calophyllum inophyllum, AM2769, TK2183. 
$\mathrm{w}^{+} \& \mathrm{~b}^{+}$Calophyllum inophyllum, AM2775, TK2190. LOC. 22A: $\mathrm{w}^{+}$Terminalia catappa, AM2709, TK2047. LOC. 22B: b" Casuarina equisetifolia L., SLS26761 (pH 5.7), SLS32222 (pH 5.4). LOC. 22C: gl ${ }^{+}$Dillenia ferruginea (Baillon) Gilg, SLS23334 (pH 5.5). ar $^{+}$Leucaena leucocephala (Lam.) de Wit., SLS26888 (pH 6.2). $a^{*}$ Vanilla planifolia Jacks. ex Andrews., SLS26781 (pH 5.6), SLS32158 (pH 6.5). LOC. 22D: $\mathrm{w}^{+}$Cinnamomum verum J. Presl, AM2744, TK2080. LOC. 22E: $\mathrm{w}^{+}$ Calophyllum inophyllum, AM2722, TK2052. LOC. 22F: $\mathrm{w}^{+}$Artocarpus heterophyllus Lam., AM2731, TK2067. LOC. 23: $\mathrm{gl}^{+}$Dillenia ferruginea ( $\mathrm{pH}$ 6.3). $\mathrm{ar}^{+}$ Lodoicea maldivica (J.F. Gmelin) Persoon, (pH 6). $\mathrm{w}^{+}$, AM2747, TK2102. LOC. 24: $\mathrm{w}^{+}$Casuarina equisetifolia, AM2762, TK2169. LOC. 25A: ar ${ }^{+}$Pandanus balfourii, SLS26831 (pH 6.1); SLS26843 (pH 6.7). LOC. 25C: ar ${ }^{+}$Passiflora foetida L., SLS26964 (pH 6.6). ar $^{+}$ Syngonium podophyllum, SLS27764 ( $\mathrm{pH} \mathrm{7).} \mathrm{LOC.}$ 27C: ar $^{+}$Cocos nucifera, SLS32194 (pH 7.9). LOC. 28A: $\mathrm{w}^{+}$Calophyllum inophyllum, AM2931, TK2479. LOC. 29: ar $^{+}$Cocos nucifera, SLS26814 (pH 5). LOC. 31A: b+ Terminalia catappa, AM2851, TK2362. LOC. 34B: $b^{+}$Cocos nucifera, AM2901, TK2436. LOC. 34C: $\mathrm{w}^{+}$Falcataria moluccana (Miq.) Barneby \& J.W. Grimes, AM2997, TK2581. LOC. 35: ar* Epipremnum pinnatum, AM3901, TK2421. LOC. 35: $\mathrm{w}^{+}$Terminalia catappa, AM2888, TK2417. LOC. 36: $\mathrm{w}^{+} \& \mathrm{~b}^{+}$ Terminalia catappa, AM3220, TK2772. LOC. 37C: b* Artocarpus heterophyllus, SLS32432 (pH 7). b* Calophyllum inophyllum, SLS26768 (pH 6). $\mathrm{b}^{+}$Chrysobalanus icaco (L.) L., SLS26935 (pH 4.6), SLS26838 (pH 4.8). LOC. 37M: $\mathrm{w}^{+}$AM2873, TK2398. LOC. 38A: $\mathrm{w}^{+}$ Cinnamomum verum, AM2904, TK2440. LOC. 38B: $\mathrm{ar}^{+}$Ipomoea sp., SLS29864 (pH 7.3). $\mathrm{ar}^{+}$Musa sp., SLS26985 (pH 6.2), SLS26676 (pH 6.3), SLS32157 (pH 6.2), SLS26361 (pH 7,4), SLS26587 (pH 7.4). LOC. 39A: ar $^{+}$Musa sp., SLS27774 (pH 7.2), SLS32150 (pH 6.9), SLS26999 (pH 7.5). $\mathrm{w}^{+}$Terminalia catappa, AM3206, TK2745. LOC. 39B: $\mathrm{w}^{+}$Calophyllum inophyllum, AM2980, AM2983, TK2552, TK2555. LOC. 39C: $\mathrm{w}^{+}$Calophyllum inophyllum, AM2973, TK2543. LOC. 40C: $\mathrm{w}^{+}$Calophyllum inophyllum, AM2921, TK2465. LOC. 41A: ar $^{+}$Cocos nucifera, SLS28564 (pH 7.1). ar $^{+}$Syngonium podophyllum, SLS29364 (pH 6.2). LOC. 42B: $\mathrm{w}^{+}$Calophyllum inophyllum, AM2942, AM2946, TK2502, TK2508. $\mathrm{w}^{+}$Calophyllum inophyllum, AM2949, TK2511. $\mathrm{w}^{+}$Calophyllum inophyllum, AM2967, TK2534. LOC. 43: $\mathrm{w}^{+} \& \mathrm{~b}^{+}$Thespesia popul- nea (L.) Sol. ex Corrêa., AM2937, AM2938, TK2490, TK2492. $\mathrm{gl}^{+}$Thespesia populnea, AM3155, TK2583. LOC. 44: $\mathrm{ar}^{+}$Leucaena leucocephala, SLS32453 (pH 6), SLS26794 (pH 6.3). LOC. 45C: ar $^{+}$Lantana camara L., SLS32327 (pH 7.2), SLS32330 (pH 6.2), SLS32456 (pH 7.1), SLS26864 (pH 7.1). LOC. 47 A: ar ${ }^{+}$Phoenicophorium borsigianum, SLS27777 (pH 4.5), SLS26798 (pH 6). LOC. 48: ar $^{+}$Cocos nucifera, SLS23874 (pH 6.2), b* Thespesia populnea, SLS26685 ( $\mathrm{pH} 7.1$ ).

A. denudata (L.) Wettst. (Granitic islands: $40 \mathrm{FC}, 3$ MC, $19 \mathrm{cp}, 8$ ps. A)

Aldabra atoll 1: gl+ Cocos sp. 3 gatherings (Ing \& Hnatiuk 1981). LOC. 3: ar* SLS26756 (pH 8.2). LOC. 6G: $\mathrm{w}^{+}$Tabebuia pallida, AM3151, TK3021. LOC. 9: $\mathrm{w}^{+}$Cinnamomum verum, AM3052, TK2803. $\mathrm{w}^{+}$Swietenia macrophylla, AM3042, AM3051, TK2790, TK2801. LOC. 20: $\mathrm{w}^{+} \& \mathrm{~b}^{+}$Calophyllum inophyllum, AM2774, TK2189. LOC. 27A: $\mathrm{w}^{+}$Terminalia catappa, AM2890, TK2423. LOC. 27B: $\mathbf{w}^{+}$Cinnamomum verum, AM3171, TK2632. LOC. 29: ar $^{+}$Cocos nucifera, SLS32502 ( $\mathrm{pH}$ 4.7). LOC. 22B: b* Casuarina equisetifolia, SLS32217 ( $\mathrm{pH}$ 5.4). LOC. 22G: $\mathrm{w}^{+} \mathrm{Ca}$ lophyllum inophyllum, AM2725, TK2059. LOC. 37A: $\mathrm{w}^{+}$Calophyllum inophyllum, AM3199, TK2742. LOC. 37C: $\mathrm{w}^{+}$\& $\mathrm{b}^{+}$Calophyllum inophyllum, AM3181, TK2700. LOC. 37G: $\mathbf{w}^{+}$Calophyllum inophyllum, AM3197, TK2734. LOC. 37K: $\mathrm{w}^{+}$Calophyllum inophyllum, AM3195, TK2669. $\mathrm{w}^{+}$Cinnamomum verum, AM3193, TK2667. LOC. 37P: $\mathrm{b}^{+}$Cinnamomum verum, AM2856, TK2370. $\mathrm{w}^{+}$Falcataria moluccana, AM2995, TK2579. LOC. 38A: $\mathrm{w}^{+} \& \mathrm{~b}^{+}$Cinnamomum verum, AM2909, TK2446. LOC. 39A: $\mathrm{w}^{+}$Terminalia catappa, AM3212, TK2746. LOC. 40C: $\mathrm{w}^{+}$Calophyllum inophyllum, AM2918, TK2460. LOC. 41B: $\mathbf{w}^{+}$ Calophyllum inophyllum, AM2970, TK2540. LOC. 45A: $\mathrm{W}^{+}$Cinnamomum verum, AM3156, TK2587.

A. helvetica (Meyl.) H. Neubert, Nowotny \& K. Baumann (Granitic islands: $1 \mathrm{MC}, 1 \mathrm{cp}, 1$ ps. S)

LOC. 17B: $\mathrm{b}^{+}$Calophyllum inophyllum, SLS324995 (pH 6.1).

A. incarnata (Pers. ex J.F. Gmel.) Pers. (Granitic islands: $1 \mathrm{MC}, 1 \mathrm{cp}, 1 \mathrm{ps} . \mathrm{R}$ )

Aldabra atoll 9: $\mathrm{w}^{+}, 1$ gathering (Ing \& Hnatiuk 1981). LOC. 45B: b* Casuarina equisetifolia, SLS32446 (pH 4.9). 
A. insignis Kalchbr. \& Cooke (Granitic islands: 14 FC, 1 MC, 7 cp, 4 ps. O)

LOC. 3: $\mathrm{w}^{+}$, AM2180, TK94S. LOC. 13: $\mathrm{b}^{+}$, AM2150, TK64S. LOC. 17: $\mathrm{w}^{+}$Plumeria alba, AM2146, TK60S. $\mathrm{w}^{+}$, AM2159, TK73S. LOC. 21F: $\mathrm{w}^{+}$ Calophyllum inophyllum, AM2768, TK2182. Loc.29: $\operatorname{ar}^{+}$Cocos nucifera, SLS32477 (pH 4.7). LOC. 39C: $\mathrm{w}^{+}$ Terminalia catappa, AM2977, TK2546. LOC. 40D: $\mathrm{w}^{+}$ Calophyllum inophyllum, AM2941, TK2499.

A. marginoundulata Nann.-Bremek. \& Y. Yamam. (Granitic islands: $1 \mathrm{MC}, 1 \mathrm{cp}, 1 \mathrm{ps} . \mathrm{S}$ )

LOC. 37D: b* Cinnamomum verum, SLS32354 (pH 5.3).

A. obvelata (Oeder) Onsberg (Granitic islands: 2 FC, $1 \mathrm{cp}, 1 \mathrm{ps} . \mathrm{R})$

LOC. 42C: $\mathrm{w}^{+} \& \mathrm{~b}^{+}$Terminalia catappa, AM2961, TK2526.

A. pomiformis (Leers) Rostaf. (Granitic islands: 2 $\mathrm{MC}, 2 \mathrm{cp}, 1$ ps. R)

LOC. 22B: $\mathrm{b}^{*}$ Casuarina equisetifolia, SLS32218 ( $\mathrm{pH}$ 5.4). LOC. 45B: $\mathrm{b}^{*}$ Casuarina equisetifolia, SLS32445 ( $\mathrm{pH} 4.9$ ).

Badhamia macrocarpa (Ces.) Rostaf. (Granitic i slands: 4 FC, 2 cp, 2 ps. R)

LOC. 42C: $\mathrm{w}^{+} \& \mathrm{~b}^{+}$Calophyllum inophyllum, AM2957, TK2522. LOC. 43: $\mathrm{b}^{+}$Thespesia populnea, AM2934, TK2484.

Ceratiomyxa fruticulosa (O.F. Müll.) T. Macbr. (Granitic islands: $12 \mathrm{FC}, 8 \mathrm{cp}, 4$ ps. O)

Aldabra atoll 9: $\mathrm{w}^{+}, 1$ gathering (Ing \& Hnatiuk 1981). LOC. 6C: $\mathrm{W}^{+}$, AM2093, TK7S. LOC. $21 \mathrm{~A}: \mathrm{W}^{+}$Terminalia catappa, AM2788, TK2208. LOC. 21 F: $\mathrm{w}^{+} \mathrm{Ca}$ lophyllum inophyllum, AM2767, TK2181. LOC. 22F: $\mathrm{w}^{+}$Cinnamomum verum. AM2742, TK2077. LOC. 31A: $\mathrm{W}^{+}$Terminalia catappa, AM2848, TK2359. LOC. 370: $\mathrm{w}^{+}$Falcataria moluccana, AM2854, TK2367. LOC. 39B: $\mathrm{w}^{+}$Terminalia catappa. LOC. 43: $\mathrm{w}^{+} \& \mathrm{~b}^{+}$ Calophyllum inophyllum, AM2933, TK2483.

C. fruticulosa var. arbuscula (Berk. \& Broome) Nann.-Bremek. (Granitic islands: 8 FC, 3 cp, 3 ps. O)

LOC. 8: $\mathrm{gl}^{+}$Phoenicophorium borsigianum, AM3098, TK2913. LOC. 37E: $\mathrm{w}^{+}$Falcataria moluc- cana, AM2990-2, TK2574. LOC. 42C: $\mathrm{w}^{+} \& \mathrm{~b}^{+}$Terminalia catappa, AM2951, AM2963, TK2514, TK2528.

Clastoderma debaryanum A. Blytt (Granitic islands: 4 MC, $2 \mathrm{cp}, 2$ ps. O)

Aldabra atoll 1, 8: gl+ Cocos sp., 5 gatherings (Ing \& Hnatiuk 1981). LOC. 29: $\operatorname{ar}^{+}$Cocos nucifera, SLS32210 (pH 4.7), SLS32213 (pH 4.5), SLS32218 ( $\mathrm{pH}$ 4.7). LOC. 31C: $\mathrm{b}^{*}$ Hernandia nymphaeifolia (Presl.) Kubitzki, SLS32488 (pH 6.9).

Collaria arcyrionema (Rostaf.) Nann.-Bremek. ex Lado (Granitic islands: $9 \mathrm{FC}, 26 \mathrm{MC}, 11 \mathrm{cp}, 8 \mathrm{ps} . \mathrm{C}$ )

LOC. 2: $\mathrm{ar}^{+}$Agave angustifolia, SLS32347 $(\mathrm{pH}$ 6.5). LOC. 3: ar*, SLS26156 (pH 8.1), SLS26157 (pH 7.5), SLS26159 (pH 7.9), SLS26425 (pH 7.7), SLS26433 (pH 8.52), SLS26720 (pH 8.4), SLS26738 (pH 7.7), SLS26744 ( $\mathrm{pH} 8.5)$, SLS26750 (pH 8.4), SLS26756 (pH 8.21), SLS26757 (pH 8.3), SLS26882 (pH 7.9), SLS26902 (pH 8.1), SLS26980 (pH 7.7). LOC. 6D: $\mathrm{ar}^{+}$Ipomoea cairica, SLS32378 (pH 6.7), SLS36778 (pH 6.9), SLS32395 (pH 6.8), SLS32398 (pH 7.1), SLS32394 (pH 7.1). LOC. 21D: $\mathrm{ar}^{+}$Epipremnum pinnatum, AM 2805. LOC. 21 B: $\mathrm{w}^{+}$Terminalia catappa, AM2789-2, TK2214. LOC. 22C: $\mathrm{b}^{+}$Calophyllum inophyllum, AM2833. LOC. 37F: $\mathrm{w}^{+}$Falcataria moluccana, AM2859, TK2374. ar* Syngonium podophyllum, SLS26760 (pH 5.6), SLS26751 (pH 5.3), SLS26758 (pH 5.8). LOC. 39A: $\mathrm{w}^{+}$Terminalia catappa, AM3207, TK2748. LOC. 40C: $\mathrm{w}^{+} \& \mathrm{~b}^{+}$Cinnamomum verum, AM2923, TK2469. LOC. 42A: $\mathrm{ar}^{+}$Cocos nucifera, SLS2673 ( $\mathrm{pH}$ 5.9). LOC. 48: $\mathrm{b}^{*}$ Thespesia populnea, SLS26731 (pH 6.1), SLS26738 (pH 7.4).

Comatricha elegans (Racib.) G. Lister (Granitic islands: $2 \mathrm{MC}, 2 \mathrm{cp}, 2 \mathrm{ps} . \mathrm{R}$ )

LOC. 19: $\mathrm{ar}^{+}$Musa sp., SLS32492 (pH 7). LOC. 37C: $\mathrm{b}^{+}$Chrysobalanus icaco, SLS32459 (pH 4.83).

C. elegans var. microspora H. Marx, in Neubert, Nowotny \& Baumann (Granitic islands: 2 FC, 1 cp, 1 ps. R)

LOC. 20: $\mathrm{w}^{+}$Calophyllum inophyllum, AM2786, TK2205.

C. laxa Rostaf. (Granitic islands: $2 \mathrm{MC}, 2 \mathrm{cp}, 2 \mathrm{ps}$. R) LOC. 23: $\mathrm{gl}^{+}$Lodoicea maldivica, SLS32431 (pH 5.6). LOC. 37C: b* Cinnamomum verum, SLS32440 (pH 6.7). 
C. pulchella (C. Bab.) (Granitic islands: 6 FC, 4 MC, 4 cp, 5 ps. O)

LOC. 6D: $\mathrm{gl}^{+}$Dianella ensifolia, SLS32466. $\mathrm{ar}^{+}$ Ipomoea cairica, SLS32471 (pH 7.1). LOC. 6G: $\mathrm{gl}^{+}$ Dillenia ferruginea, AM3137, TK2990. gl ${ }^{+}$Pandanus balfourii, AM3138, TK2993. LOC. 37C: ar $^{+}$Cinnamomum verum, SLS32454 (pH 6), SLS32458 (pH 4.6). LOC. 42C: $\mathrm{gl}^{+}$Calophyllum inophyllum. AM2959, TK2523.

C. tenerrima (M.A. Curtis) G. Lister (Granitic islands: $1 \mathrm{MC}, 1 \mathrm{cp}, 1$ ps. S)

LOC. 48: $\mathrm{b}^{*}$ Thespesia populnea, SLS32207 (pH 7.2).

*Craterium concinnum Rex (Granitic islands: 2 FC, 1 cp, 1 ps. R)

LOC. 42C: $\mathrm{gl}^{+}$Terminalia catappa, AM2956, TK2520.

\section{C. leucocephalum (Pers. ex J.F. Gmel.) Ditmar (R)}

Aldabra atoll 14: $\mathrm{gl}^{+}$Mystroxylon sp., 2 gatherings (Ing \& Hnatiuk 1981).

C. minutum var. brunneum (Nann.-Bremek.) L.G. Krieglst. (Granitic islands: 4 FC, 2 cp, 1 ps. R)

LOC. 34B: $\mathrm{gl}^{+}$Terminalia catappa, AM2898, TK2433. LOC. 35: $\mathrm{w}^{+} \& \mathrm{~b}^{+}$Terminalia catappa, AM2884, TK2414.

Cribraria aurantiaca Schrad. (Granitic islands: 2 FC, 1 cp, 1 ps, Fig. 2b. R)

LOC. 37F: $\mathrm{W}^{+}$Falcataria moluccana, AM2860, TK2376.

C. cancellata (Batsch) Nann.-Bremek. (Granitic islands: 6 FC, 2 cp, 3 ps. O)

LOC. 37-l: $\mathrm{w}^{+} \& \mathrm{~b}^{+}$Calophyllum inophyllum, AM3185, TK2674. $\mathrm{w}^{+} \& \mathrm{~b}^{+}$Cinnamomum verum, AM3183, TK2673. LOC. 39A: $\mathrm{W}^{+}$Terminalia catappa, AM3203, TK2749.

C. confusa Nann.-Bremek. \& Y. Yamam. (Granitic islands: $2 \mathrm{MC}, 2 \mathrm{cp}, 2$ ps. R)

LOC. 23: $\mathrm{b}^{*}$ Erythroxylum sechellarum $\mathrm{O}$. Schulz, SLS32414 (pH 4.2). LOC. 37C: $\mathrm{b}^{+}$Chrysobalanus icaco, SLS32348 (pH 4.6).
C. intricata Schrad. (Granitic islands: $6 \mathrm{FC}, 2 \mathrm{cp}$, 3 ps. O)

LOC. 9: $\mathrm{w}^{+} \& \mathrm{~b}^{+}$Cinnamomum verum, AM3057, TK2810, $\mathrm{w}^{+}$Swietenia macrophylla, AM3048, TK2797. LOC. 42C: $\mathrm{w}^{+}$Calophyllum inophyllum, AM2963, TK2529.

C. intricata var. dictydioides (Cooke \& Balf.f.) Lister (Granitic islands: 24 FC, $10 \mathrm{cp}, 6$ ps. C)

LOC. 6D: $\mathrm{w}^{+}$Calophyllum inophyllum, AM3119, TK2942. LOC. 6E: $\mathrm{w}^{+}$Cinnamomum verum, AM3074, TK2838. LOC. 16: $\mathrm{gl}^{+}$Cocos nucifera, AM2161, TK75S. LOC. 17: $\mathrm{w}^{+}, \mathrm{AM} 2145$, TK59S. $\mathrm{w}^{*}$ Nephrosperma vanhoutteanum Balf., AM2165, TK79S. LOC. 31 A: $\mathrm{w}^{+}$Terminalia catappa, AM2850, TK2360. LOC. 37B: $\mathrm{w}^{+} \mathrm{Ca}$ lophyllum inophyllum, AM3179, TK2680. LOC. 37-1: $\mathrm{w}^{+}$Calophyllum inophyllum, AM3177, TK2676. LOC. 37I: $\mathrm{w}^{+}$Falcataria moluccana, AM2989, TK2567. LOC. 39C: $\mathrm{w}^{+}$Terminalia catappa, AM2976, TK2545. LOC. 45A: $\mathrm{w}^{+}$Calophyllum inophyllum, AM3157, TK2590. $\mathrm{w}^{+}$Cinnamomum verum, AM3157, TK2589.

\section{C. languescens $\operatorname{Rex}(\mathrm{R})$}

Aldabra atoll 8, 9: $\mathrm{gl}^{+} \operatorname{Cocos} \mathrm{sp} ., \mathrm{b}^{+}, 2$ gatherings (Ing \& Hnatiuk 1981).

C. lepida Meyl. (Granitic islands: 6 FC, 3 cp, 3 ps. O) LOC. 22A: fungi on $\mathrm{w}^{+}$Terminalia catappa, AM2711, TK2050. LOC. 43: $\mathrm{w}^{+} \& \mathrm{~b}^{+}$Thespesia populnea, AM2936, TK2487. LOC. 45A: $\mathrm{w}^{+} \& \mathrm{~b}^{+}$Calophyllum inophyllum, AM3158, TK2592.

C. microcarpa (Schrad.) Pers. (Granitic islands: 4 FC, 5 MC, 7 cp, 5 ps. O)

Aldabra atoll 1: $\mathrm{gl}^{+}$Cocos sp., 1 gathering (Ing \& Hnatiuk 1981). LOC. 8: b* Tabebuia pallida, SLS32407 (pH 6.2). LOC. 9: $\mathrm{gl}^{+}$Deckenia nobilis, SLS32384 (pH 5.3). LOC. 22B: b* Calophyllum inophyllum, SLS32403 (pH 5.7). LOC. 22G: $\mathrm{w}^{+}$Calophyllum inophyllum, AM2726, TK2060. LOC. 23: gl ${ }^{+}$Dillenia ferruginea, SLS32486 (pH 6.3). LOC. 36: $\mathrm{w}^{+} \& \mathrm{~b}^{+}$ Terminalia catappa, AM3217, TK2774. LOC. 37C: b* Calophyllum inophyllum, SLS32336 (pH 6.6).

C. pachydictyon Nann.-Bremek. (Granitic islands: 2 FC, 1 cp, 1 ps. R)

LOC. 6G: $\mathrm{w}^{+}$, AM3134, TK2986. 
C. tenella Schrad. (Granitic islands: $2 \mathrm{FC}, 1 \mathrm{cp}, 1 \mathrm{ps} . \mathrm{R}$ ) LOC. 40C: $\mathrm{w}^{+}$Cinnamomum verum, AM2925, TK2471.

C. violacea Rex (Granitic islands: $18 \mathrm{MC}, 11 \mathrm{cp}$, 10 ps. C)

Aldabra atoll 8, 15: $\mathrm{gl}^{+} \operatorname{Cocos}$ sp., $\mathrm{b}^{+}, 2$ gatherings (Ing \& Hnatiuk 1981). LOC. 6A: b* Tabebuia pallida, SLS32424 (pH 6.2). LOC. 6D: ar $^{+}$Ipomoea cairica, SLS32428 (pH 7.1). LOC. 22D: b* Artocarpus heterophyllus, SLS32226 (pH 7.33). LOC. 22E: b* Syzygium jambos L. (Alston), SLS32427 (pH 6.1). LOC. 25B: $b^{*}$ Cordia subcordata, SLS32208 ( $\mathrm{pH} 7.2$ ). LOC. 25C: $\mathrm{ar}^{+}$Passiflora foetida, SLS32205 (pH 7.8). LOC. 26: b* Terminalia catappa, SLS32408 (pH 6.6), SLS32420 (pH 6.6). LOC. 37C: b* Calophyllum inophyllum, SLS32429 (pH 7.4). LOC. 45D: b* Cordia subcordata, SLS32402 (pH 5.6), SLS32325 (pH 6.6), SLS32338 (pH 6.6), SLS32340 (pH 6.6). LOC. 47 B: b* Rhizophora mucronata, SLS32351 (pH 7.1). LOC. 48: $\mathrm{b}^{*}$ Thespesia populnea, SLS32444 (pH 7.2), SLS32206 (pH 7.1), SLS32583 (pH 7.2), SLS32358 (pH 7.4).

Diachea bulbillosa (Berk. \& Broome) Lister (Granitic islands: 6 FC, 3 cp. O)

Aldabra atoll 1, 3: $\mathrm{gl}^{+}$Mystroxylon sp., Sideroxylon sp., Tournefortia sp., Tricalysia sp., 2 gatherings (Ing \& Hnatiuk 1981). LOC. 6C: developed from yellow plasmodium on $\mathrm{gl}^{+}$Plumeria alba, AM2091, TK5S. LOC. 13: $\mathrm{gl}^{+}$, AM2153, TK67S. LOC. 18: $\mathrm{gl}^{+}$ Plumeria alba, AM2106, TK20S.

D. leucopodia (Bull.) Rostaf. (Granitic islands: 6 FC, $1 \mathrm{MC}, 3 \mathrm{cp}, 4$ ps. O)

Aldabra atoll 1, 4: $\mathrm{gl}^{+}$Casuarina equisetifolia, Lumnitzera sp., Mystroxylon sp., Terminalia catappa, 3 gatherings (Ing \& Hnatiuk 1981). LOC. 2: $\mathrm{gl}^{+}$ Tabebuia pallida, AM3088, TK2882. $\mathrm{gl}^{+}$Artocarpus altilis (Parkinson) Fosberg, AM3085, TK2878. LOC. 18: $\mathrm{gl}^{+}$Plumeria alba, AM2105, TK19S. LOC. 37C: $\mathrm{ar}^{+}$ Cinnamomum verum, SLS32339 (pH 4.6).

D. radiata G. Lister \& Petch (R)

Aldabra atoll 1: $\mathrm{gl}^{+}$Pemphis sp., Tournefortia sp., 2 gatherings (Ing \& Hnatiuk 1981).

Dictydiaethalium dictyosporum (Schumach.) Rostaf. (Granitic islands: 2 FC, 1 cp, 1 ps. R)
LOC. 39C: $\mathrm{w}^{+}$\& $\mathrm{b}^{+}$Terminalia catappa, AM2978, TK2547.

Diderma chondrioderma (de Bary \& Rostaf.) Kuntze (Granitic islands: 22 FC, 10 MC, 15 cp, 11 ps, Fig. 2f. C)

LOC. 2: gl ${ }^{+}$Artocarpus altilis, AM3077, TK2864. LOC. 7: ar $^{+}$Cocos nucifera, AM2112, TK26S. LOC. 8: moss on $\mathrm{b}^{*}$ Tabebuia pallida, AM3106, TK2923. moss on $b^{*}$ Phoenicophorium borsigianum. AM3097, TK2912. LOC. 11: moss on b* Artocarpus altilis, AM2133, TK47S. moss on $\mathrm{b}^{*}$ Artocarpus altilis with Physarum pusillum, AM2136, TK50S. LOC. 12: moss on $\mathrm{b}^{*}$, AM2120, TK34S. LOC. 22C: ar $^{+}$Calophyllum inophyllum, SLS32209 (pH 4.8). LOC. 22B: b* Calophyllum inophyllum, SLS32221 ( $\mathrm{pH}$ 6.4). b* Casuarina equisetifolia, SLS32451 (pH 5.7), SLS32224 (pH 5.4). LOC. $21 \mathrm{~F}$ : moss on $\mathrm{b}^{*}$ Calophyllum inophyllum, AM2773, TK2188. LOC. 29: b* Calophyllum inophyllum, SLS32429 (pH 5.7). LOC. 33: moss on b* Cocos nucifera, AM2932, TK2481. LOC. 37C: $\mathrm{b}^{+}$Chrysobalanus icaco, SLS32426 ( $\mathrm{pH}$ 5.7). b* Cinnamomum verum, SLS32412 (pH 6.7). LOC. 38B: b* Artocarpus altilis, SLS32412 (pH 5.7). LOC. 42C: moss on b* Calophyllum inophyllum, AM2954, TK2517. LOC. 43: moss on $\mathrm{b}^{*}$ Thespesia populnea, AM2934, TK2485. LOC. 48: b* Calophyllum inophyllum, SLS32212 (pH 5.9). $b^{*}$ Rhizophora mucronata, AM2834.

D. effusum (Schwein.) Morgan (Granitic islands: 32 FC, 19 MC, 24 cp, 13 ps. A)

Aldabra atoll 1, 5, 10, 12, 13: gl ${ }^{+}$Acalypha sp., Maytenus sp., Mystroxylon sp., Pemphis sp., Sideroxylon sp., Tarenna supra-axillaris, Tricalysia sp., 12 gatherings (Ing \& Hnatiuk 1981). LOC. 6A: gl ${ }^{+} \mathrm{Dil}-$ lenia suffruticosa (Griff ex Hook.f. \& Thomson) Martelli, AM3060, TK2816. LOC. 6C: gl ${ }^{+}$, AM2089, TK3S. LOC. 6D: $\mathrm{gl}^{+}$Calophyllum inophyllum, AM3115, TK2937. LOC. 6E: ar* Nephrolepis biserrata on Elaeis guineensis, SLS32332 (pH 6.4). $\mathrm{b}^{*}$ Tabebuia pallida, SLS32369 (pH 6.4). LOC. 6F: gl ${ }^{+}$, AM3065, TK2824. LOC. 7: On fallen small twig, AM2113, TK27S. LOC. 8: gl ${ }^{+}$Tabebuia pallida, AM3103, TK2918. LOC. 18: b $^{*}$, AM2097, TK11S. LOC. 21E: gl ${ }^{+}$Adenanthera pavonina L., SLS32368 (pH 5.7), AM2806. LOC. 22C: $\mathrm{ar}^{+}$Alstonia macrophylla, SLS32355 (pH 6). gl ${ }^{+}$Phoenicophorium borsigianum, SLS32401 (pH 5.3). LOC. 22F: gl ${ }^{+}$Calophyllum inophyllum, AM2732, TK2068. 
LOC. 23: $\mathrm{gl}^{+}$Lodoicea maldivica, SLS32763 (pH 5.5). $\mathrm{gl}^{+}$Lodoicea maldivica, SLS32323 (pH 5.1). LOC. 25A: ar $^{+}$Cocos nucifera, AM2757, TK2132. LOC. 25B: $\mathrm{b}^{*}$ Cordia subcordata, SLS32765 (pH 5.8). LOC. 27A: $\mathrm{gl}^{+}$Calophyllum inophyllum, AM2896, TK2429. LOC. 31 A: $b^{+}$Cocos nucifera, AM2847, TK2358. LOC. 34B: $\mathrm{gl}^{+}$Terminalia catappa, AM2899, TK2434. LOC. 35 : $\mathrm{gl}^{+}$Terminalia catappa, AM2887, TK2416. LOC. 37H: $\mathrm{gl}^{+}$Cinnamomum verum, AM2864, AM2868, TK2389, TK2392. LOC. 38B: ar $^{+}$Cocos nucifera, SLS32169 (pH 6.4), SLS32170 (pH 6.2). LOC. 39B: gl ${ }^{+} \& \mathrm{~b}^{+}$Calophyllum inophyllum, AM2985, TK2557. LOC. 45B: b* Casuarina equisetifolia, SLS32485 (pH 6.7), SLS32489 (pH 7). LOC. 45D: b* Cordia subcordata, SLS32344 (pH 6.6), SLS32352 (pH 6.7). LOC. 48: ar $^{+}$Cocos nucifera, SLS32480 (pH 6.3), SLS32345 (pH 6.2), SLS32351 (pH 6.2). $\mathrm{b}^{*}$ Calophyllum inophyllum AM2830.

D. hemisphaericum (Bull.) (Granitic islands: $6 \mathrm{MC}$, $5 \mathrm{cp}, 5$ ps. O)

Aldabra atoll 2, 10: $\mathrm{gl}^{+}$Acalypha sp., 2 gatherings (Ing \& Hnatiuk 1981). LOC. 2: $a^{+}$Epipremnum pinnatum, SLS32288 (pH 5.8). LOC. 37C: ar $^{+}$Cinnamomum verum, SLS32328 (pH 6). LOC. 38B: b* Artocarpus altilis, SLS32213 (pH 5.7), SLS32195 (pH 5.6). LOC. 39A: ar ${ }^{+}$Syngonium podophyllum, SLS32359 (pH 8.5). LOC. 48: $\mathrm{gl}^{+}$Cocos nucifera, SLS32371 (pH 5).

D. rimosum Eliasson \& Nann.-Bremek. (Granitic islands: 4 FC, 2 cp, 1 ps. R)

LOC. 6A: gl ${ }^{+}$, AM3063, TK2821. LOC. $37 \mathrm{H}: \mathrm{gl}^{+}$ Calophyllum inophyllum, AM2863, TK2386.

D. saundersii (Berk. \& Broome ex Massee) E. Sheld. (Granitic islands: $11 \mathrm{FC}, 1 \mathrm{MC}, 5 \mathrm{cp}, 4$ ps, Fig. 2c-d. O)

LOC. 6E: moss on $b^{*}$ Cinnamomum verum, AM3075, TK2839. LOC. 8: $\mathrm{gl}^{+}$, AM3108, TK2927, $\mathrm{gl}^{+}$, AM3109, TK2929. LOC. 39A: $\mathrm{b}^{+}$Cocos nucifera, AM3208, TK2751. LOC. 39B: gl $^{+}$Cocos nucifera, AM2987, TK2562. LOC. 40B: $\mathrm{b}^{+}$Calophyllum inophyllum, AM3903, TK2458.

Didymium anellus Morgan (Granitic islands: $3 \mathrm{MC}$, $3 \mathrm{cp}, 3$ ps. R)

Aldabra atoll 13: gl ${ }^{+}$Sideroxylon sp., 1 gathering (Ing \& Hnatiuk 1981). LOC. 22C: ar* Cassytha filiformis L. over-growing on Prosopis juliflora, SLS32467 (pH 5.9). LOC. 39A: gl ${ }^{+}$Alocasia macror- rhiza, SLS32381 (pH 6.6). LOC. 48: gl+ Cocos nucifera, SLS32482 (pH 5.6).

D. bahiense Gottsb. (Granitic islands: 2 FC, 2 MC, 3 cp, 3 ps. R)

LOC. 20: ar $^{+}$Falcataria moluccana, SLS32497 (pH 6.2). LOC. 42C: gl ${ }^{+}$Terminalia catappa, AM2943, TK2503. LOC. 48: gl ${ }^{+}$Cocos nucifera, SLS32356 (pH 5).

D. clavus (Alb. \& Schwein.) Rabenh. (Granitic islands: 2 FC, $1 \mathrm{cp}, 1 \mathrm{ps} . \mathrm{R}$ )

LOC. 6G: $\mathrm{gl}^{+}$Cinnamomum verum, AM3135, TK2988.

D. columellacavum Hochg., Gottsb. \& Nann.Bremek. (Granitic islands: $24 \mathrm{FC}, 9$ cp, 6 ps. C)

LOC. 2: $\mathrm{gl}^{+}$Cinnamomum verum, AM3083, TK2874. LOC. 6A: ar* palm, AM3061, TK2818. LOC. 6D: gl ${ }^{+}$Artocarpus altilis, AM3117, TK2940. LOC. 6F: b* Nephrosperma vanhoutteanum, AM3070, TK2834. LOC. 6G: $\mathrm{gl}^{+}$Pandanus balfourii, AM3149, TK3018. $\mathrm{gl}^{+}$, AM3146, AM3147, AM3148, TK3007, TK3011, TK3015. LOC. 8: $\mathrm{gl}^{+}$, AM3107, TK2925. LOC. 22F: $\mathrm{w}^{+}$ Cinnamomum verum, AM2743, TK2078. LOC. 30: $\mathrm{gl}^{+}$ Mangifera indica L., AM2879, TK2407. LOC. 42C: gl $^{+}$ Calophyllum inophyllum, AM2966, TK2532.

D. dubium Rostaf. (Granitic islands: $1 \mathrm{MC}, 1 \mathrm{cp} . \mathrm{S}$ ) LOC. 3: $b^{*}$, SLS26429 (pH 8.3).

\section{D. eximium Peck (S)}

Aldabra atoll 7: gl $^{+}$Mystroxylon sp., 1 gathering (Ing \& Hnatiuk 1981).

Didymium floccosum G.W. Martin, K.S. Thind \& Rehill (S)

Aldabra atoll 1: b* Cocos sp., 1 gathering (Ing \& Hnatiuk 1981).

\section{D. intermedium J. Schröt. (R)}

Aldabra atoll 1, 4, 16: $\mathrm{w}^{+} \& \mathrm{gl}^{+}$Tricalysia sp., 4 gatherings (Ing \& Hnatiuk 1981).

D. iridis (Ditmar) Fr. (Granitic islands: $16 \mathrm{MC}, 9 \mathrm{cp}$, 10 ps. O)

LOC. 19: $\operatorname{ar}^{+}$Musa sp., SLS32474 (pH 6.9). LOC. 22C: gl ${ }^{+}$Dillenia ferruginea, SLS32478 (pH 5.5), $\mathrm{ar}^{+}$ Leucaena leucocephala, SLS32437 (pH 6.4). ar* Va- 
nilla planifolia, SLS32171 (pH 6.2). LOC. 23: $\mathrm{ar}^{+}$Lodoicea maldivica, SLS32452 (pH 6.6), SLS32493 (pH 6.5). LOC. 25A: $\operatorname{ar}^{+}$Pandanus balfourii, SLS32481 (pH 6.4). LOC. 37C: $\mathrm{ar}^{+}$Cinnamomum verum, SLS32462 (pH 6). LOC. 38B: gl $^{+}$Calophyllum inophyllum, SLS32466 (pH 7.9). LOC. 39A: $\mathrm{ar}^{+}$Musa sp., SLS32421 (pH 6.9); SLS32431 (pH 7.2), SLS32439 (pH 7.5). LOC. 45C: ar $^{+}$Lantana camara, SLS32464 (pH 6.2). LOC. 50: $\mathrm{gl}^{+}$Cocos nucifera, SLS32318 (pH 6.6), SLS32324 (pH 6.5), SLS32328 (pH 6.9).

\section{D. leoninum Berk. \& Broome (R)}

Aldabra atoll 9: $\mathrm{w}^{+} \& \mathrm{gl}^{+}, 2$ gathering (Ing \& Hnatiuk 1981).

\section{D. melanospermum (Pers.) T. Macbr. (S)}

Aldabra atoll 4: gl ${ }^{+}$Solanum nigrum L., 1 gathering (Ing \& Hnatiuk 1981).

D. minus (Lister) Morgan (Granitic islands: $2 \mathrm{FC}$, $1 \mathrm{MC}, 1 \mathrm{cp}, 2 \mathrm{ps}$. R)

Aldabra atoll 1: gl ${ }^{+}$Maytenus sp., Tricalysia sp., 1 gathering (Ing \& Hnatiuk 1981). LOC. 6D: $\mathrm{gl}^{+} \mathrm{Di}$ anella ensifolia, SLS32328 (pH 6). gl ${ }^{+}$Dillenia ferruginea, AM3116, TK2938.

D. nigripes (Link) Fr. (Granitic islands: 14 FC, 2 MC, $6 \mathrm{cp}, 4$ ps. C)

Aldabra atoll 1, 3, 9: gl ${ }^{+}, 4$ gatherings (Ing \& Hnatiuk 1981). LOC. 3: b*, SLS26753 (pH 8.1). LOC. 6G: $\mathrm{gl}^{+}$Dillenia ferruginea, AM3126, AM3136, TK2972, TK2989. $\mathrm{gl}^{+}$Pandanus balfourii, AM3144, TK3000. LOC. 8: $\mathrm{gl}^{+}$Artocarpus altilis, AM3113, TK2932. LOC. 10A: b* $^{*}$ AM2138, TK52S. LOC. 15: $\mathrm{w}^{+}$, AM2127, TK41S. b*, AM2128, TK42S. LOC. 22C: $a^{+} \&$ ar* Vanilla planifolia, SLS32154 (pH 7).

D. ochroideum G. Lister (Granitic islands: $2 \mathrm{FC}$, $7 \mathrm{MC}, 3 \mathrm{cp}, 2$ ps. O)

LOC. 2: $\operatorname{ar}^{+}$Cordyline fruticosa (L.) A. Chev., SLS32392 (pH 8.6). LOC. 3: b* , SLS26429 (pH 8.3), SLS26721 (pH 7.7), SLS26736 (pH 7.4), SLS26755 (pH 7.9), SLS26758 (pH 7.9), SLS26763 (pH 7.7). LOC. 34B: $b^{+}$Alocasia macrorrhizos (L.) G. Don, AM2903, TK2438.

D. ovoideum Nann.-Bremek. (Granitic islands: 2 FC, $1 \mathrm{cp}, 1 \mathrm{ps} . \mathrm{R})$
LOC. 6F: gl ${ }^{+}$Calophyllum inophyllum, AM3068, TK2828.

D. squamulosum (Alb. \& Schwein.) Fr. \& Palmquist (Granitic islands: 6 FC, $12 \mathrm{MC}, 10 \mathrm{cp}, 13$ ps. C)

Aldabra atoll 1, 2, 6: gl ${ }^{+}$Cocos sp., Maytenus sp., Sideroxylon sp., 8 gatherings (Ing \& Hnatiuk 1981). LOC. 2: $\mathrm{ar}^{+}$Agave angustifolia, SLS32320 (pH 7.2), $\mathrm{ar}^{+}$ Cordyline fruticosa, SLS32325 (pH 7.2). gl ${ }^{+}$Terminalia catappa, AM3078, TK2866. LOC. 3: b*, SLS26736 (pH 7.4). LOC. 6A: gl ${ }^{+}$Dillenia suffruticosa, AM3059, TK2814. LOC. 6D: gl $^{+}$Dianella ensifolia, SLS32330 (pH 6.4). LOC. 6E: ar* Ipomoea cairica, SLS32340 (pH 6.8), ar* Nephrolepis biserrata on Elaeis guineensis, SLS32321 ( $\mathrm{pH}$ 6.4). ar* Thunbergia grandiflora, SLS32334 (pH 6.4). LoC. 17: gl ${ }^{+}$Plumeria alba, AM2164, TK78S. LOC. 22C: ar ${ }^{+}$Leucaena leucocephala, SLS32391 ( $\mathrm{pH}$ 6.5). LOC. 25C: ar $^{+}$Syngonium podophyllum, SLS32313 ( $\mathrm{pH}$ 6.9). LOC. 38B: $\mathrm{gl}^{+} \mathrm{Ca}$ lophyllum inophyllum, SLS32166 (pH 7.7), SLS32167 (pH 8.2). LOC. 48: $\mathrm{gl}^{+}$Cocos nucifera, SLS32356 (pH 5).

D. verrucosporum A.L. Welden (Granitic islands: 2 FC, 1 cp, 1 ps. R)

Aldabra atoll 4: $\mathrm{gl}^{+}$Mystroxylon sp., 1 gathering (Ing \& Hnatiuk 1981). LOC. 42C: gl ${ }^{+}$Calophyllum inophyllum, AM2959, TK2524.

Echinostelium minutum de Bary (Granitic islands: $10 \mathrm{MC}, 9 \mathrm{cp}, 9$ ps. O)

LOC. 8: ar $^{+}$Roscheria melanochaetes $\mathrm{H}$. Wendl. SLS32411 (pH 4.8) LOC. 23: b* Erythroxylum sechellarum, SLS32421 ( $\mathrm{pH}$ 4.2). LOC. 26: $\mathrm{b}^{*}$ Terminalia catappa, SLS32406 (pH 6.6). LOC. 37D: b* Cinnamomum verum, SLS32385 ( $\mathrm{pH}$ 5). b* Falcataria mollucana, SLS32422 (pH 6.9). LOC. 38B: b* Artocarpus altilis, SLS32214 (pH 5.7). LOC. 42A: b* Calophyllum inophyllum, SLS32373 (pH 5.5). LOC. 45B: $\mathrm{b}^{*}$ Casuarina equisetifolia, SLS32430 ( $\mathrm{pH} 4.9$ ). LOC. 46: $\mathrm{b}^{*}$ Calophyllum inophyllum, SLS32413 (pH 5.5). LOC. 47 A: ar $^{+}$ Phoenicophorium borsigianum, SLS32346 ( $\mathrm{pH}$ 5.6).

E. paucifilum K.D. Whitney (Granitic islands: $2 \mathrm{MC}$, $2 \mathrm{cp}, 2$ ps. R)

LOC. 24: b* Tabebuia pallida, SLS32437 ( $\mathrm{pH}$ 5.7). LOC. 48: b* Calophyllum inophyllum, SLS32439 (pH 5.9). 
Fuligo cinerea (Schwein.) Morgan (Granitic islands: $2 \mathrm{MC}, 1 \mathrm{cp} . \mathrm{R})$

LOC. 3: $b^{*}$, SLS26738 (pH 7.7), SLS26161 (pH 8.4).

\section{F. septica (L.) F.H. Wigg. (R)}

Aldabra atoll 1, 8: $\mathrm{w}^{+}$Casuarina sp., gl ${ }^{+}$Sporobolus sp., 4 gatherings (Ing \& Hnatiuk 1981).

F. septica var. candida (Pers.) R.E. Fr. (Granitic islands: $8 \mathrm{FC}, 4 \mathrm{cp}, 3$ ps. O)

LOC. 2: $\mathrm{w}^{+}$Cinnamomum verum, AM3082, TK2872. LOC. 3: ar $^{+}$, AM2172-1, TK86S. LOC. 23: gl $^{+}$ Lodoicea maldivica, AM2748, TK2103. LOC. 36: $\mathrm{w}^{+}$\& $\mathrm{b}^{+}$Terminalia catappa, AM3214, TK2775.

F. septica var. flava (Pers.) Lázaro Ibiza (Granitic islands: $8 \mathrm{FC}, 4 \mathrm{cp}, 3$ ps. O)

LOC. 22B: $\mathrm{w}^{*} \& \mathrm{~b}^{*}$ Thespesia populnea, AM2707, TK2028. LOC. 31 A: $\mathrm{w}^{+}$Terminalia catappa, AM2845, TK2355. LOC. 31 B: $\mathrm{w}^{+}$Terminalia catappa, AM2852, TK2364. LOC. 40D: $\mathrm{w}^{+}$Calophyllum inophyllum, AM2947, TK2500.

Hemitrichia calyculata (Speg.) M.L. Farr (Granitic islands: $50 \mathrm{FC}, 20 \mathrm{cp}, 5 \mathrm{ps}$. A)

Aldabra atoll 1, 8, 9: $\mathrm{gl}^{+} \operatorname{Cocos} \mathrm{sp} ., 10$ gatherings (Ing \& Hnatiuk 1981). LOC. 2: $\mathrm{w}^{+}$, AM3086, TK2879. LOC. 3: $b^{*}$, AM2177, TK91S. LOC. 6E: moss on $\mathrm{w}^{+}$ Cinnamomum verum, AM3076, TK2844. LOC. 6G: $\mathrm{w}^{+}$, AM3132, TK2983. LOC. 20: $\mathrm{w}^{+}$Calophyllum inophyllum, AM2784, TK2204. LOC. 21C: $\mathrm{w}^{+}$Falcataria moluccana, AM2764, TK2174. LOC. 22E: $\mathrm{w}^{+}$Calophyllum inophyllum, AM2724, TK2053. LOC. 22F: $\mathrm{w}^{+}$ Cinnamomum verum, AM2738, TK2073. LOC. 30: $\mathrm{w}^{+}$, AM2882, TK2410. LOC. 31 A: $\mathrm{w}^{+}$Terminalia catappa, AM2846, TK2357. LOC. 35: $\mathrm{w}^{+}$Terminalia catappa, AM2889, TK2418. LOC. 36: $\mathrm{w}^{+}$Terminalia catappa, AM3221, TK2776. LOC. 37B: $\mathrm{w}^{+}$Calophyllum inophyllum, AM3181, AM3188, AM3191, AM3196, TK2682, TK2683, TK2684, TK2685. LOC. 37I: $\mathbf{w}^{+}$ Falcataria moluccana, AM2990, TK2569. LOC. 37P: $\mathrm{w}^{+}$Falcataria moluccana, AM2855, TK2369. LOC. 38A: $\mathrm{w}^{+}$Cinnamomum verum, AM2905, TK2441. LOC. 39A: $\mathrm{b}^{+}$Cocos nucifera, AM3209, TK2754. $\mathrm{w}^{+}$ Terminalia catappa, AM3202, TK2752. LOC. 40A: $\mathrm{W}^{+}$ Terminalia catappa, AM2912, TK2450. $\mathrm{w}^{+} \& \mathrm{~b}^{+}$Terminalia catappa, AM2915, TK2453. LOC. 40C: $\mathrm{w}^{+}$\& $\mathrm{b}^{+}$Calophyllum inophyllum, AM2921, TK2466. LOC.
42C: $\mathrm{w}^{+}$Calophyllum inophyllum, AM2970, TK2535.

H. minor G. Lister (Granitic islands: $4 \mathrm{MC}, 3 \mathrm{cp}$, 4 ps. R)

LOC. 22B: b* Calophyllum inophyllum, SLS32220 ( $\mathrm{pH}$ 6.4). $\mathrm{b}^{*}$ Casuarina equisetifolia, SLS32223 (pH 5.4). LOC. 24: $\mathrm{b}^{*}$ Tabebuia pallida, SLS32443 ( $\mathrm{pH}$ 5.7). LOC. 37C: $b^{*}$ Cinnamomum verum, SLS32416 (pH 6.7).

H. serpula (Scop.) Rostaf. ex Lister (Granitic islands: $24 \mathrm{FC}, 1 \mathrm{MC}, 12 \mathrm{cp}, 5$ ps. C)

Aldabra atoll 1: gl ${ }^{+}$Cocos sp., 1 gathering (Ing \& Hnatiuk 1981). LOC. 7: b* Cocos nucifera, AM2117, TK31S. LOC. 8: $\mathrm{gl}^{+}$Pandanus balfourii, AM3104, TK2920. LOC. 10A: ar* Cocos nucifera, AM2124, TK38S. LOC. 19: b* Phoenicophorium borsigianum, AM2746, TK2086. LOC. 22B: $\operatorname{ar}^{+}$Cocos nucifera, AM2708, TK2029. LOC. 25A: ar $^{+}$Cocos nucifera, AM2752, TK2124. LOC. 34B: $b^{+}$Cocos nucifera, AM2902, TK2437. LOC. 37C: ar $^{+}$Cinnamomum verum, SLS32400 (pH 6). LOC. 39A: $\mathrm{ar}^{+} \& \mathrm{~b}^{*} \operatorname{Cocos} n u-$ cifera, AM3211, TK2755. LOC. 42A: $\mathrm{w}^{+}$Calophyllum inophyllum, AM3163, TK2627. LOC. 45A: $\mathrm{w}^{+}$Calophyllum inophyllum, AM3159, TK2594. $\mathrm{ar}^{+}$Cocos nucifera, AM3160, TK2595. LOC. 49: $\mathrm{gl}^{+}$Cocos nucifera, AM2717, TK2239.

Lamproderma scintillans (Berk. \& Broome) Morgan (Granitic islands: $14 \mathrm{MC}, 6 \mathrm{cp}, 7$ ps. O)

LOC. 2: ar $^{+}$Agave angustifolia, SLS26418 (pH 7.0). LOC. 3:b*, SLS26422 (pH 7.3), SLS26160 (pH 8.53). LOC. 6E: ar $^{+}$Ipomoea cairica, SLS26461 (pH 7.1). $\mathrm{ar}^{+}$ Thunbergia grandiflora, SLS26168 (pH 6.4). LOC. 21 E: $\mathrm{ar}^{+}$Epipremnum pinnatum, SLS26171 (pH 7.4). LOC. 38B: gl $^{+}$Calophyllum inophyllum, SLS32181 (pH 7.7), SLS32195 (pH 8.2), SLS32176 (pH 6.3), SLS32178 (pH

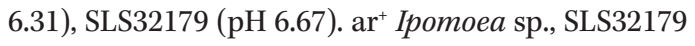
$\left(\mathrm{pH}\right.$ 8.5). LOC. 41A: $\mathrm{ar}^{+}$Syngonium podophyllum, SLS26169 (pH 6.2), SLS32183 (pH 6.2).

Licea biforis Morgan (Granitic islands: $1 \mathrm{MC}, 1 \mathrm{cp}$, 1 ps. S)

LOC. 48: b* Calophyllum inophyllum, SLS32417 (pH 5.9).

L. kleistobolus G.W. Martin (Granitic islands: 1 MC, $1 \mathrm{cp}, 1$ ps. S)

LOC. 26: $\mathrm{b}^{*}$ Terminalia catappa, SLS32411 (pH 6.6). 
L. minima Fr. (Granitic islands: $1 \mathrm{MC}, 1 \mathrm{cp}, 1 \mathrm{ps} . \mathrm{S}$ )

LOC. 22E: b* Syzygium jambos, SLS32418 (pH 6.1).

L. operculata (Wingate) G.W. Martin (Granitic islands: $2 \mathrm{MC}, 2$ loc, 2 ps. R)

LOC. 37C: b* Falcataria mollucana, SLS32409 (pH 6.9). LOC. 48: $\mathrm{b}^{*}$ Thespesia populnea, SLS32198 (pH 7.2).

L. rufocuprea Nann.-Bremek. \& Y. Yamam. (Granitic islands: $1 \mathrm{MC}, 1 \mathrm{cp}, 1$ ps. S)

LOC. 48: $\mathrm{b}^{*}$ Thespesia populnea, SLS32985 (pH 7.2).

L. scyphoides T.E. Brooks \& H.W. Keller (Granitic islands: $1 \mathrm{MC}, 1 \mathrm{cp}, 1 \mathrm{ps}$. S)

LOC. 23: b* Erythroxylum sechellarum, SLS32448 (pH 4.2).

Lycogala epidendrum (L.) Fr. (Granitic islands: 16 FC, 8 cp, 4 ps. O)

Aldabra atoll 1: ar $^{+}$Agave sp., 1 gathering (Ing \& Hnatiuk 1981). LOC. 6C: $\mathrm{w}^{+}$, AM2087, TK1S. LOC. 22G: $\mathrm{w}^{+}$Artocarpus heterophyllus, AM2730, TK2065. LOC. 27B: $\mathrm{w}^{+}$Calophyllum inophyllum, AM3174, TK2635. LOC. 37I: $\mathrm{w}^{+}$Falcataria moluccana, AM2988-1, TK2566. LOC. 39A: $\mathrm{w}^{+}$Tabebuia pallida, AM3201, TK2757. LOC. 41A: $\mathrm{w}^{+}$Calophyllum inophyllum, AM2782, TK2339. LOC. 41 B: $\mathrm{w}^{+}$Calophyllum inophyllum, AM2968, TK2538. LOC. 42A: $\mathrm{w}^{+} \mathrm{Ca}$ lophyllum inophyllum, AM3161, TK2628.

L. exiguum Morgan (Granitic islands: 4 FC, $2 \mathrm{cp}, 2 \mathrm{ps}$. R)

LOC. 41 B: $\mathrm{w}^{+}$Calophyllum inophyllum, AM2969, TK2539. LOC. 49: $\mathrm{w}^{+}$Cocos nucifera, AM2716, TK2238.

Macbrideola decapillata H.C. Gilbert (Granitic islands: $3 \mathrm{MC}, 2 \mathrm{cp}, 2 \mathrm{ps}$. R)

LOC. 9: b* Swietenia macrophilla, SLS32442 (pH 6.6). LOC. 22B: $b^{*}$ Casuarina equisetifolia, SLS32436 (pH 5.7), SLS32216 (pH 5.4).

M. scintillans H.C. Gilbert (Granitic islands: 1 MC, $1 \mathrm{cp}, 1$ ps. S)

LOC. 47B: b* Rhizophora mucronata, SLS32393 (pH 7.5).
Metatrichia vesparia (Batsch) Nann.-Bremek. ex G.W. Martin \& Alexop. (R)

Aldabra atoll 1, 8, 15: $\mathrm{gl}^{+}$Cocos sp., 5 gatherings (Ing \& Hnatiuk 1981).

Perichaena chrysosperma (Curr.) Lister (Granitic islands: $16 \mathrm{MC}, 13 \mathrm{cp}, 10 \mathrm{ps}$. O)

LOC. 2: $\mathrm{gl}^{+}$Cheilocostus speciosus, SLS32447 (pH 7). LOC. 3: b* SLS26164 (pH 7.8). LOC. 6D: $\mathrm{ar}^{+}$ Dioscorea sp., SLS32329 (pH 7.1). LOC. 17 B: b b Calo- $^{+}$ phyllum inophyllum, SLS32491 (pH 6.5). LOC. 20: ar* Syngonium podophyllum, SLS32340 (pH 7.7). LOC. 22C: ar $^{+}$Leucaena leucocephala, SLS32498 (pH 6.2). LOC. 22D: b* Artocarpus heterophyllus, SLS32227 (pH 7.3). LOC. 27B: b* Calophyllum inophyllum, SLS32598 (pH 7), ar+ Cocos nucifera, SLS32530 (pH 7.9). LOC. 38B: ar $^{+}$Ipomoea sp., SLS32531 (pH 7.3). LOC. 39A: ar ${ }^{+}$Syngonium podophyllum, SLS32385 (pH 8.7). LOC. 45D: b* Cordia subcordata, SLS32321 (pH 6.7), SLS32345 (pH 6.6), SLS32349 (pH 6.6). LOC. 48: b* Thespesia populnea, SLS32200 (pH 7.1). LOC. 50: b* Cordia subcordata, SLS32419 (pH 6.7).

P. corticalis (Batsch) Rostaf. (Granitic islands: $2 \mathrm{FC}$, $1 \mathrm{cp} . \mathrm{R})$

Aldabra atoll $1: \mathrm{w}^{+}, 1$ gathering (Ing \& Hnatiuk 1981). LOC. 10A: gl ${ }^{+}$Cocos nucifera, AM2126, TK40S.

P. depressa Lib. (Granitic islands: 6 FC, 10 MC, 10 cp, 9 ps. O)

Aldabra atoll 16: $\mathrm{w}^{+}, 1$ gathering (Ing \& Hnatiuk 1981). LOC. 2: $\mathrm{w}^{+} \& \mathrm{~b}^{+}$Cinnamomum verum, AM3081, TK2870. gl ${ }^{+} \&$ ar $^{+}$Phoenicophorium borsigianum, SLS32350 ( $\mathrm{pH}$ 5.7). LOC. 6D: $\mathrm{ar}^{+}$Dioscorea sp., SLS32371 (pH 7.1). LOC. 24: $\mathrm{w}^{+}$Cordia subcordata. AM2760, TK2167. LOC. 37C: ar $^{+}$Cinnamomum verum, SLS32381 (pH 6.4). LOC. 37M: $\mathrm{w}^{+}$. AM2874, TK2399. LOC. 38B: $\mathrm{gl}^{+}$Calophyllum inophyllum, SLS32189 (pH 7.9). LOC. 39A: ar $^{+}$Musa sp., SLS32134 (pH 7.5). LOC. 45C: $\operatorname{ar}^{+}$Lantana camara, SLS32170 (pH 7.2). LOC. 45D: b* Cordia subcordata, SLS32156 (pH 6.6). LOC. 48: gl ${ }^{+}$Cocos nucifera, SLS32360 (pH 6.8). $\mathrm{b}^{*}$ Thespesia populnea, SLS32140 ( $\mathrm{pH}$ 6.9), SLS32175 (pH 7.1).

P. dictyonema Rammeloo (Granitic islands: $18 \mathrm{MC}$, $5 \mathrm{cp}, 4$ ps. O)

LOC. 3: $\mathrm{b}^{*}$, SLS26156 (pH 8.1), SLS26160 (pH 
8.5), SLS26161 (pH 8.4), SLS26423 (pH 7.6), SLS26424 (pH 7.9), SLS26429 (pH 8.3), SLS26485 (pH 8.0), SLS26723 (pH 7.5), SLS26724 (pH 8.4), SLS26725 (pH 8.1), SLS26745 (pH 8.5), SLS26750 (pH 8.4), SLS26753 (pH 8.1), SLS26757 (pH 8.3). LOC. 25C: ar $^{+}$ Syngonium podophyllum, SLS32483 (pH 6.9). LOC. 38B: gl $^{+}$Calophyllum inophyllum, SLS32193 (pH 8.2). LOC. 39A: ar $^{+}$Musa sp., SLS32374 (pH 6.9). LOC. 41 A: ar $^{+}$Cocos nucifera, SLS32177 (pH 6.8).

P. pedata (Lister \& G. Lister) G. Lister ex E. Jahn (Granitic islands: $3 \mathrm{MC}, 2 \mathrm{cp}, 3 \mathrm{ps} . \mathrm{R}$ )

LOC. 6D: b* Dieffenbachia sequine, SLS32372 (pH 7.7). ar $^{+}$Dioscorea sp. SLS32389 (pH 6.9). LOC. 22C: $\mathrm{gl}^{+}$Adenanthera pavonina, SLS32354 (pH 6.4).

P. quadrata T.Macbr. (Granitic islands: $2 \mathrm{FC}, 1 \mathrm{cp}$, 1 ps. R)

LOC. 7: $\mathrm{b}^{+}$Cocos nucifera, AM2119, TK33S.

P. vermicularis (Schwein.) Rostaf. (Granitic islands: $12 \mathrm{MC}, 8 \mathrm{cp}, 9$ ps. O)

LOC. 3:b*, SLS26725 (pH 8.1). LOC. 6E: $\mathrm{ar}^{+} I p-$ omoea cairica, SLS32368 (pH 6.8). ar* Nephrolepis biserrata on Elaeis guineensis, SLS32365 (pH 6.4). ar $^{+}$Thunbergia grandiflora, SLS32461 (pH 6.4). LOC. 22C: ar $^{+}$Vanilla planifolia, SLS32139 (pH 7). LOC. 25A: ar ${ }^{+}$Pandanus balfourii, SLS32471 (pH 6.4). LOC. 25C: ar* Passiflora foetida, SLS32128 (pH 8.1). LOC. 38B: $\mathrm{ar}^{+}$Musa sp., SLS32182 (pH 6.3). LOC. 41 A: $\mathrm{ar}^{+}$ Cocos nucifera, SLS32148 (pH 7.1), SLS32167 (pH 6.8). LOC. 44: $\mathrm{ar}^{+}$Leucaena leucocephala, SLS32182 (pH 6.3), SLS32461 (pH 6.4).

Physarella oblonga (Berk. \& M.A. Curtis) Morgan (Granitic islands: $6 \mathrm{FC}, 2 \mathrm{cp}, 2 \mathrm{ps} . \mathrm{O}$ )

LOC. 37P: $\mathrm{b}^{+}$Thespesia populnea, AM2857, TK2371. $\mathrm{w}^{+} \& \mathrm{~b}^{+}$, AM2875, TK2400. LOC. 49: $\mathrm{w}^{+}$Cocos nucifera, AM2719, TK2241.

Physarum aeneum (Lister) R.E. Fr. (Granitic islands: $1 \mathrm{MC}, 1 \mathrm{cp}, 1 \mathrm{ps} . \mathrm{S}$ )

LOC. 22D: b* Artocarpus heterophyllus, SLS32225 (pH 7.3).

P. album (Bull.) Chevall. (Granitic islands: $8 \mathrm{FC}$, $2 \mathrm{MC}, 6 \mathrm{cp}, 3$ ps. O)

Aldabra atoll 1, 8 (as Physarum nutans Pers.): $\mathrm{w}^{+}$
\& gl+ Cocos sp., 2 gatherings (Ing \& Hnatiuk 1981). LOC. 3: moss on $b^{*}$ Artocarpus altilis, AM2171, TK85S. LOC. 9: $\mathrm{w}^{+}$Swietenia macrophylla, AM3044, TK2793. LOC. 11 : moss on b*, AM2141, TK55S. LOC. 23: $\mathrm{gl}^{+}$Lodoicea maldivica, SLS32411 (pH 5.6). LOC. 29: ar $^{+}$Cocos nucifera, SLS32499 (pH 4.7). LOC. 49: $\mathrm{w}^{+}$Cocos nucifera, AM2718, TK2240.

P. atroviolaceum G. Moreno, Y. Yamam. \& A. Castillo (Granitic islands: $2 \mathrm{FC}, 1 \mathrm{cp}, 1 \mathrm{ps}$. R)

LOC. 6G: $\mathrm{gl}^{+}$Pandanus balfourii, AM3142, TK2997.

P. auriscalpium Cooke (Granitic islands: 2 FC, $1 \mathrm{cp}$, 1ps. R)

LOC. 7: b* Cocos nucifera, AM2108, TK22S.

P. bethelii T. Macbr. ex G. Lister (Granitic islands: 2 FC, 1 cp, 1 ps. R)

LOC. 8: $\mathrm{w}^{+}$Phoenicophorium borsigianum, AM3099, TK2914.

P. bogoriense Racib. (Granitic islands: $12 \mathrm{FC}, 5 \mathrm{cp}$, 4 ps. O)

Aldabra atoll 1, 3, 5, 9, 10, 11: moss on rocks, $\mathrm{gl}^{+}$Mystroxylon sp., Sideroxylon sp., Tricalysia sp., 7 gatherings (Ing \& Hnatiuk 1981). LOC. 8: $\mathrm{gl}^{+}$ Tabebuia pallida, AM3105, TK2921. LOC. $37-\mathrm{l} \mathrm{gl}^{+}$ Cinnamomum verum, AM3178, TK2677. LOC. 37G: $\mathrm{gl}^{+}$Calophyllum inophyllum, AM2862, TK2380. $\mathrm{gl}^{+}$ Cinnamomum verum, AM2869, TK2393. LOC. 37M: $\mathrm{gl}^{+}$Cinnamomum verum, AM2871, TK2396. LOC. 39A: gl ${ }^{+}$Terminalia catappa, AM3213, TK2759.

P. cinereum (Batsch) Pers. (Granitic islands: $2 \mathrm{MC}$, $1 \mathrm{cp}, 1$ ps, Fig. 2e. R)

Aldabra atoll 1: gl ${ }^{+}$Carica papaya, 1 gathering (Ing \& Hnatiuk 1981). LOC. 35: ar* Epipremnum pinnatum, AM3899, TK2419.

P. compressum Alb. \& Schwein. (Granitic islands: 6 FC, 39 MC, $11 \mathrm{cp}, 10$ ps. A)

Aldabra atoll 1: gl+ Agave sp., 1 gathering (Ing \& Hnatiuk 1981). LOC. 2: ar $^{+}$Epipremnum pinnatum, SLS32360 (pH 6.9). LOC. 3: gl+, AM2174, TK88S. b*, SLS26735 (pH 7.6), SLS26152 (pH 7.6), SLS26156 (pH 8.1), SLS26158 (pH 8.0), SLS26160 (pH 8.5), SLS26161 (pH 8.4), SLS26163 (pH 7.6), SLS26164 
(pH 7.8), SLS26420 (pH 7.6), SLS26422 (pH 7.3), SLS26423 (pH 7.6), SLS26425 (pH 7.7), SLS26428 (pH 7.4), SLS26430 (pH 7.6), SLS26485 (pH 8.0), SLS26723 (pH 7.5), SLS26725 (pH 8.1), SLS26746 ( $\mathrm{pH} 7.7$ ), SLS26757 ( $\mathrm{pH} 8.3$ ), SLS26882 ( $\mathrm{pH} 7.9$ ), SLS26748 (pH 7.2). LOC. 6E: ar $^{+}$Ipomoea cairica, SLS32136 (pH 6.4), SLS32146 (pH 7.7). LOC. 13: gl AM2156, TK70S. LOC. 20: ar $^{+}$Falcataria moluccana, SLS32131 ( $\mathrm{pH}$ 6.5). ar* Syngonium podophyllum, SLS32144 (pH 7.8), SLS32146 (pH 7.7). LOC. 22C: $\mathrm{ar}^{+}$Calophyllum inophyllum, SLS32358 ( $\mathrm{pH}$ 6.9). $\mathrm{gl}^{+}$Phoenicophorium borsigianum, SLS32145 $(\mathrm{pH}$ 5.9). ar* Vanilla planifolia, SLS32159 (pH 6.5). Loc. 23: ar* Martellidendron hornei, SLS32482 (pH 5.9). LOC. 25C: ar* Passiflora foetida, SLS32152 (pH 6.4),

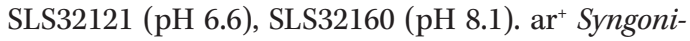
um podophyllum, SLS32155 (pH 6.9). LOC. 35: ar* Epipremnum pinnatum, AM3900, AM3902, TK2420. LOC. 37J: gl ${ }^{+}$Alocasia macrorrhiza, SLS32132 (pH 6.5). LOC. 38B: ar ${ }^{+}$Ipomoea sp., SLS32143 (pH 7.3). $\mathrm{ar}^{+}$Syngonium podophyllum, SLS32164 (pH 6.3).

P. crateriforme Petch (Granitic islands: 14 FC, $19 \mathrm{MC}, 15 \mathrm{cp}, 11$ ps. C)

Aldabra atoll 9: algae on steep rock faces, 3 gatherings (Ing \& Hnatiuk 1981). LOC. 1: b*, AM2166, TK80S. LOC. 3: Tremella on $\mathrm{w}^{+}$Gleditsia triacanthos, AM2184, TK98S. b*, SLS26159 (pH 7.9), SLS26485 (pH 8.0), SLS26725 (pH 8.1), SLS26735 (pH 7.6), SLS26753 (pH 8.1), SLS26741 (pH 6.7), SLS26741 (pH 6.7). LOC. 4: moss on b* Tamarindus indica L., AM2169, TK83S. LOC. 6E: b* Tabebuia pallida, SLS32364 (pH 6.4). $\mathrm{ar}^{+}$ Thunbergia grandiflora, SLS32363 (pH 6.8). LOC. 11 : $\mathrm{w}^{+}$Cocos nucifera, AM2140, TK54S. LOC. 15: $\mathrm{b}^{+}$Cocos nucifera, AM2139, TK53S. LOC. 17: moss on $\mathrm{b}^{*}$ Acacia concinna (Willd.) DC. (mimosa), AM2163, TK77S. LOC. 19: $\mathrm{ar}^{+}$Cocos nucifera, (pH 7). LOC. 22E: b* Syzygium jambos, SLS32433 (pH 6.1). LOC. 36: b* Calophyllum inophyllum \& Terminalia catappa \& Cordia subcordata, SLS32370 (pH 6.8). LOC. 37C: b* Calophyllum inophyllum, SLS32319 (pH 7). LOC. 38B: b* Artocarpus altilis, SLS32372 (pH 5.7). ar $^{+}$Ipomoea sp., SLS32151 (pH 8.5). LOC. 43: $\mathrm{w}^{*} \& \mathrm{~b}^{*}$ Thespesia populnea, AM2938, TK2494. LOC. 48: b* Calophyllum inophyllum, SLS32423 (pH 5.9). b* Thespesia populnea, SLS32141 (pH 6.9), SLS32138 (pH 7.3), SLS32142 (pH 7.2). LOC. 50: b* Cordia subcordata, SLS32441 (pH 6.7).
P. cremiluteum Y.F. Chen \& C.H. Liu (Granitic islands: 24 FC, 8 cp, 6 ps, Fig. 2a. C)

LOC. 2: gl ${ }^{+}$Tabebuia pallida, AM3087, TK2880. $\mathrm{w}^{+}$Cinnamomum verum, AM3079, TK2867. $\mathrm{gl}^{+}$ Tabebuia pallida, AM3084, TK2876. LOC. 6F: gl ${ }^{+}$Dillenia suffruticosa, AM3069, TK2831. gl ${ }^{+}$Mangifera indica, AM3066, TK2826. LOC. 21 D: gl ${ }^{+}$Cinnamomum verum \& Falcataria moluccana \& Tabebuia pallida, AM2765, TK2176. LOC. 28A: $\mathrm{gl}^{+}$Calophyllum inophyllum, AM2930, TK2478. LOC. 34B: gl ${ }^{+}$Terminalia catappa, AM2900, TK2435. LOC. 35: gl ${ }^{+}$Terminalia catappa, AM2885, TK2415. LOC. 39B: gl ${ }^{+}$Calophyllum inophyllum, AM2981, TK2558. LOC. 42B: $\mathrm{gl}^{+}$ Terminalia catappa, AM2950, AM2956, TK2512, TK2521.

P. decipiens M.A. Curtis (Granitic islands: $3 \mathrm{MC}$, 2 cp, 2 ps. R)

LOC. 3:b*, SLS26872 (pH 8.3). LOC. 48: b* Calophyllum inophyllum, SLS32435 ( $\mathrm{pH}$ 5.9). $\mathrm{b}^{*}$ Thespesia populnea, SLS32190 (pH 6.1).

\section{P. dictyosporum G.W. Martin (S)}

Aldabra atoll 12: $\mathrm{gl}^{+}, 1$ gathering (Ing \& Hnatiuk 1981).

P. didermoides (Pers.) Rostaf. (Granitic islands: $2 \mathrm{MC}, 1 \mathrm{cp} . \mathrm{R}$ )

LOC. 3: b* SLS26757 (pH 8.3), SLS26764 (pH 8.2).

P. echinosporum Lister (Granitic islands: $2 \mathrm{FC}, 1 \mathrm{cp}$, 1 ps. O)

Aldabra atoll 1, 4, 7: $\mathrm{gl}^{+}$Carica papaya, Cocos nucifera, Lumnitzera racemosa, Terminalia catap$p a, 5$ gatherings (Ing \& Hnatiuk 1981). LOC. 22A: $\mathrm{w}^{+}$ Terminalia catappa, AM2710, TK2049.

P. flavicomum Berk. (Granitic islands: 2 FC, 1 cp. R) LOC. 17: moss on $b^{+}$, AM2157, TK71S.

P. florigerum (Meyl.) Y. Yamam. (Granitic islands: 2 FC, 1 cp. R)

LOC. 8: $\mathrm{w}^{+}$palm, AM3112, TK2931.

\section{P. globuliferum (Bull.) Pers. (O)}

Aldabra atoll 1, 9, 16: $\mathrm{gl}^{+} \operatorname{Cocos} \mathrm{sp} ., \mathrm{w}^{+}$Casuarina sp., mangrove, 17 gatherings (Ing \& Hnatiuk 1981). 
P. gyrosum Rostaf. (Granitic islands: $5 \mathrm{MC}, 4 \mathrm{cp}$, 4 ps. R)

LOC. 2: $\operatorname{ar}^{+}$Phoenicophorium borsigianum, SLS32351 (pH 5.7). LOC. 25C: ar Syngonium podophyllum, SLS32736 (pH 6.3), SLS32745 (pH 7). LOC. 38B: gl $^{+}$Calophyllum inophyllum, SLS32781 (pH 8.2). LOC. 41A: ar $^{+}$Cocos nucifera, SLS32163 (pH 7.2).

P. hongkongense Chao H. Chung (Granitic islands: 16 FC, $2 \mathrm{MC}, 10 \mathrm{cp}, 6$ ps. O)

LOC. 4: moss on $b^{*}$ Tamarindus indica, AM2168, TK82S. LOC. 6C: gl $^{+}$, AM2092, TK6S. LOC. 6F: $\mathrm{gl}^{+}$Terminalia catappa, AM3064, TK2822. LOC. 9: $\mathrm{gl}^{+}$Swietenia macrophylla, AM3049, TK2799. LOC. 13: $\mathrm{gl}^{+}, \mathrm{AM} 2147$, TK61S. $\mathrm{gl}^{+}$with Physarum mutabile, AM2149, TK63S. LOC. 18: gl ${ }^{+}$, AM2098, TK12S. LOC. 27A: gl ${ }^{+}$Calophyllum inophyllum, AM2894, TK242. LOC. 30: $\mathrm{gl}^{+}$Mangifera indica, AM2880, TK2409. LOC. 37C: b* Artocarpus heterophyllus, SLS32438 (pH 7). LOC. 45D: b* Cordia subcordata, SLS32343 (pH 6.6).

P. javanicum Racib. (Granitic islands: $1 \mathrm{MC}, 1 \mathrm{cp} . \mathrm{S}$ ) LOC. $3: b^{*}$, SLS26761 (pH 7.5).

P. lakhanpalii Nann.-Bremek. \& Y. Yamam. (Granitic islands: $15 \mathrm{FC}, 10 \mathrm{MC}, 10 \mathrm{cp}, 6$ ps. C)

LOC. 2: $\mathrm{w}^{+}$Tabebuia pallida, AM3093, TK2888. LOC. 3: ar" Musa paradisiaca L., AM2173, TK87S. $\mathrm{b}^{*}$, SLS26752 (pH 7.6). LOC. 7: ar* Cocos nucifera, AM2109, TK23S. ar* Cocos nucifera, AM2111. ar* Cocos nucifera, AM2115, TK29S. LOC. 12: $\mathrm{w}^{+}$Plumeria alba, AM2121, TK35S. ar $^{+}$Cocos nucifera, $\mathrm{w}^{+}$palm with Arcyria cinerea, AM2122, TK36S. LOC. 17B: $\mathrm{b}^{+}$ Calophyllum inophyllum, SLS32476 (pH 6), SLS32381 (pH 6.1). LOC. 22B: b* Calophyllum inophyllum, SLS32219 (pH 6.4). LOC. 23: $\mathrm{ar}^{+}$Lodoicea maldivica, SLS32371 (pH 6.6). LOC. 25A: ar $^{+}$Pandanus balfourii, SLS32762 (pH 6.7). ar $^{+}$Cocos nucifera, AM2753, TK2126. LOC. 29: ar $^{+}$Cocos nucifera, SLS32355 (pH 5), SLS32362 ( $\mathrm{pH}$ 6). LOC. 50: gl ${ }^{+}$Cocos nucifera, SLS32388 (pH 6.4), SLS32399 (pH 6.6).

\section{P. leucopus Link (S)}

Aldabra atoll 1: moss-covered rock, 1 gathering (Ing \& Hnatiuk 1981).

P. luteolum Peck (Granitic islands: 2 FC, 1 cp, 1 ps. R)
LOC. 27 A: gl ${ }^{+}$Calophyllum inophyllum, AM2895, TK2428.

P. melleum (Berk. \& Broome) Massee (Granitic islands: $49 \mathrm{FC}, 10 \mathrm{MC}, 25 \mathrm{cp}, 17 \mathrm{ps}$. A)

Aldabra atoll 4: $\mathrm{gl}^{+}$Sideroxylon sp., 1 gatherin (Ing \& Hnatiuk 1981). LOC. 2: gl ${ }^{+}$Tabebuia pallida, AM3087, TK2881. w+ Cinnamomum verum, AM3079, TK2868. LOC. 3: b*, SLS26874 (pH 7.1). LOC. 5: ar $^{+}$ Agave, AM2175, TK89S. LOC. 6D: gl ${ }^{+}$Dianella ensifolia, SLS26882 (pH 6). LOC. 6F: gl ${ }^{+}$, AM3067, TK2827. LOC. 6G: gl ${ }^{+}$Cinnamomum verum, AM3143, TK2999. $\mathrm{gl}^{+}$Dillenia ferruginea, AM3139, TK2995. LOC. 8: $\mathrm{gl}^{+}$Tabebuia pallida, AM3114, TK2934. LOC. 13: $\mathrm{gl}^{+}$, AM2152, TK66S. LOC. 15: gl ${ }^{+}$, AM2142, TK56S. LOC. 18: gl+, AM2099, AM2101, AM2102, AM2103, TK13S, TK15S, TK16S. LOC. 22C: ar $^{+}$Leucaena leucocephala, (pH 5.8). $\mathrm{gl}^{+}$Phoenicophorium borsigianum, SLS26835 (pH 5.9). LOC. 25A: $\operatorname{ar}^{+}$Cocos nucifera, AM2756, TK2131. LOC. 27A: gl ${ }^{+}$Calophyllum inophyllum, AM2892, TK2425. LOC. 29: $\operatorname{ar}^{+}$Cocos nucifera, SLS26824 (pH 6). LOC. 30: gl Mangifera indica, AM2878, TK2406. LOC. 35: Xylaria sp. on $\mathrm{w}^{+}$, AM2883, TK2412. LOC. 36: gl ${ }^{+}$Terminalia catappa, AM3223, TK2777. LOC. 37C: ar $^{+}$Cinnamomum verum, SLS32353 (pH 6), SLS32178 (pH 6.44). ar* Phymatodes scolopendria, SLS32380 (pH 6.3). LOC. 37J: ar* Syngonium podophyllum, SLS32331 (pH 5.8). $\mathrm{gl}^{+}$Calophyllum inophyllum, AM2862, TK2381. $\mathrm{gl}^{+}$Cinnamomum verum, AM2867, TK2391. LOC. 37K: $\mathrm{gl}^{+}$Dicranopteris linearis (Burm.f.) Underw., AM3184, TK2671. LOC. 37M: gl ${ }^{+}$Cheilocostus speciosus, AM2870, TK2395. LOC. 38B: b* Artocarpus altilis, SLS32211 (pH 5.7). ar $^{+}$Cocos nucifera, AM2781, TK2295, SLS32178 (pH 6.4), (pH 6.3). ar $^{+}$Musa sp., (pH 6.3), SLS32187 (pH 7.4). ar $^{+}$Syngonium podophyllum, ( $\mathrm{pH}$ 6.3). LOC. 39B: $\mathrm{W}^{+}$Terminalia catappa, AM2982, TK2554. LOC. 40A: $\mathrm{w}^{+}$Terminalia catappa, AM2913, TK2451. LOC. 47A: $\mathrm{ar}^{+}$Phoenicophorium borsigianum, SLS32196 (pH 5.9), SLS32189 (pH 6).

P. melleum f. luteum Y.Yamam. (Granitic islands: 2 FC, 1 cp. R)

LOC. 18: gl+, AM2100, TK14S.

P. mutabile (Rostaf.) G. Lister (Granitic islands: 6 FC, 3 cp, 1 ps. O)

Aldabra atoll 1, 3, 4, 5: b* \& $\mathrm{gl}^{+}$Cyperus sp., $\mathrm{gl}^{+}$ 
Mystroxylon sp. \& Ochna sp., 5 gatherings (Ing \& Hnatiuk 1981). LOC. 2: gl ${ }^{+}$Tabebuia pallida, AM3089, TK2884. LOC. 13: $\mathrm{gl}^{+}$with P. hongkongense, AM 2149, TK63S. LOC. 18: gl ${ }^{+}$, AM2096, TK10S.

\section{P. nicaraguense $\mathrm{T}$. Macbr. (R)}

Aldabra atoll 3, 14: moss-covered rock, moss on $\mathrm{w}^{+}$Ochna sp., 2 gatherings (Ing \& Hnatiuk 1981).

P. notabile T. Macbr. (Granitic islands: 2 FC, $1 \mathrm{cp}$, 1 ps. R)

LOC. 2: $\mathrm{ar}^{+}$Epipremnum pinnatum, AM3080, TK2869.

P. nucleatum Rex (Granitic islands: $4 \mathrm{FC}, 2 \mathrm{cp}, 2$ ps. R) LOC. 22F: $\mathrm{W}^{+}$palm, AM2741, TK2076; LOC. 36: $\mathrm{w}^{+} \& \mathrm{~b}^{+}$Terminalia catappa, AM3219, TK2778.

P. oblatum T. Macbr. (Granitic islands: $11 \mathrm{MC}, 5 \mathrm{cp}$, 4 ps. O)

Aldabra atoll 1: $\mathrm{gl}^{+}$Cocos sp, 1 gathering (Ing \& Hnatiuk 1981). LOC. 3: b*, SLS26738 (pH 7.7), SLS26160 (pH 8.5), SLS26420 (pH 7.6), SLS26433 (pH 8.5), SLS26484 (pH 7.7). LOC. 6E: b* Tabebuia pallida, SLS32367 (pH 6.4). LOC. 21E: $\mathrm{ar}^{+}$Epipremnum pinnatum, SLS32147 (pH 7.4), SLS32151 (pH 7.5). LOC. 47A: ar $^{+}$Phoenicophorium borsigianum, SLS32165 (pH 4.9). LOC. 48: $\mathrm{b}^{*}$ Thespesia populnea, SLS32137 (pH 6.1), SLS32425 (pH 7.4).

P. plicatum Nann.-Bremek. \& Y. Yamam. (Granitic islands: $8 \mathrm{FC}, 1 \mathrm{cp}, 4$ ps. O)

LOC. 6G: $\mathrm{gl}^{+}$Cinnamomum verum, AM3131, TK2979. $\mathrm{gl}^{+}$Deckenia nobilis, AM3128, TK2975. $\mathrm{gl}^{+}$ Dillenia ferruginea, AM3122, TK2967. gl ${ }^{+}$Pandanus balfourii, AM3145, TK3002.

P. pusillum (Berk. \& M.A. Curtis) G. Lister (Granitic islands: $7 \mathrm{FC}, 26 \mathrm{MC}, 17 \mathrm{cp}, 16$ ps. C)

Aldabra atoll 1: $\mathrm{gl}^{+}$Cocos sp, 1 gathering (Ing \& Hnatiuk 1981). LOC. 2: ar $^{+}$Agave angustifolia, SLS32391 ( $\mathrm{pH}$ 8.6). gl ${ }^{+}$Cheilocostus speciosus, SLS32431 ( $\mathrm{pH}$ 7). LOC. 10A: moss on b* Artocarpus altilis with Diderma chondrioderma, AM2136, TK50S. LOC. 16: $\mathrm{gl}^{+}$Cocos nucifera, AM2160, TK74S. LOC. 19: ar $^{+}$Cocos nucifera, SLS32485 (pH 4.9). ar $^{+}$ Musa sp., SLS32469 (pH 7.3), SLS32471 (pH 7.2), SLS32498 (pH 7.4). LOC. 20: ar $^{+}$Falcataria moluc- cana, SLS32155 (pH 6.2), SLS32496 (pH 6.5). LOC. 21E: $\mathrm{gl}^{+}$Adenanthera pavonina, SLS32511 (pH 5.8). LOC. 22B: ar* Cassytha filiformis over-growing Prosopis juliflora, SLS32515 ( $\mathrm{pH}$ 5.9). LOC. 22C: ar $^{+}$Alstonia macrophylla, SLS32422 (pH 6). $\mathrm{ar}^{+}$Leucaena leucocephala, SLS32496 (pH 6.5). ar* Vanilla planifolia, SLS32398 (pH 5.6). LOC. 25A: ar ${ }^{+}$Pandanus balfourii, SLS32434 (pH 6.7). LOC. 27 B: gl ${ }^{+}$Cocos nucifera, AM3164, TK2636, TK2637. LOC. 37J: ar* Syngonium podophyllum, SLS32521 (pH 8.3). LOC. 38B: $\mathrm{ar}^{+}$Syngonium podophyllum, SLS32531 (pH 6.3), SLS32168 (pH 6.3). LOC. 41A: ar $^{+}$Cocos nucifera, SLS32525 (pH 6.8). ar $^{+}$Syngonium podophyllum, SLS32155 (pH 6.2), SLS32174 (pH 6.2). LOC. 45C: ar Lantana $^{+}$ camara, SLS32463 (pH 7). LOC. 46: ar* Ficus lutea Vahl, SLS32189 (pH 6). LOC. 47A: ar ${ }^{+}$Phoenicophorium borsigianum, SLS32123 (pH 4.3), SLS32167 (pH 4.5). LOC. 48: $\mathrm{gl}^{+}$Cocos nucifera, SLS32516 (pH 6.9), SLS325332 (pH 6.8).

P. roseum Berk. \& Broome (Granitic islands: $10 \mathrm{FC}$, $1 \mathrm{MC}, 6 \mathrm{cp}, 5$ ps. O)

Aldabra atoll 1, 8: $\mathrm{gl}^{+}$Cocos sp, 3 gatherings (Ing \& Hnatiuk 1981). LOC. 2: gl ${ }^{+}$Cinnamomum verum, AM3095, TK2890. LOC. 3: lichen on b* Spondias cytherea, AM2170, TK84S.LOC. 6F: $\mathbf{w}^{+}$, AM3072, TK2837. LOC. 8: b* Nephrosperma vanhoutteanum, AM3096, TK2910. LOC. 37C: $\mathrm{w}^{+}$Calophyllum inophyllum, AM3200, TK2698. LOC. 44: $\operatorname{ar}^{+}$Leucaena leucocephala, SLS32503 (pH 4.4).

\section{P. serpula Morgan (O)}

Aldabra atoll 1, 9, 12, 13: $\mathrm{b}^{*} \& \mathrm{gl}^{+}$Agave sp., Cocos sp., Mystroxylon sp., Sideroxylon sp., in an old nest of a tropic bird Phaethron sp., 8 gatherings (Ing \& Hnatiuk 1981).

P. sessile Brândza (Granitic islands: 12 FC, 6 cp, 2 ps. O)

LOC. 6A: gl ${ }^{+}$Calophyllum inophyllum, AM3058, TK2812. LOC. 7: ar* Cocos nucifera, AM2116, TK30S. LOC. 13: $\mathrm{gl}^{+}$, AM2148, TK62S. LOC. 27A: $\mathrm{gl}^{+}$Calophyllum inophyllum, AM2891, TK2424. LOC. 37 B: $\mathrm{gl}^{+}$ Cinnamomum verum, AM3186, TK2686. LOC. 37M: $\mathrm{gl}^{+}$Cinnamomum verum. AM2872, TK2397.

P. stellatum (Massee) G.W. Martin (Granitic islands: 25 FC, 10 cp, 4 ps. C)

Aldabra atoll $1: \mathrm{gl}^{+}$Cocos sp., $\mathrm{b}^{+}$mangrove, 
5 gatherings (Ing \& Hnatiuk 1981). LOC. 2: $\mathrm{w}^{+}$ Tabebuia pallida, AM3091, TK2887. LOC. 3: $\mathrm{gl}^{+}$, AM2178, TK92S. LOC. 6A: $\mathbf{w}^{+}$, AM3062, TK2819. LOC. 10B: ar $^{+}$Cocos nucifera, AM3040, TK2788. LOC. 14: $\mathrm{b}^{+} \& \mathrm{w}^{+}$, AM2144, TK58S. LOC. 15: $\mathrm{b}^{+}$Cocos nucifera, AM2130, TK44S. LOC. 27B: $\mathrm{w}^{+} \& \mathrm{~b}^{+}$Calophyllum inophyllum, AM3173, TK2639. LOC. 31 B: $\mathrm{ar}^{+}$Cocos nucifera, AM2853, TK2365. LOC. 42B: $\mathrm{w}^{+}$ $\& \mathrm{~b}^{+}$Terminalia catappa, AM2945, TK2505. $\mathrm{w}^{*} \& \mathrm{~b}^{*}$ Calophyllum inophyllum, AM2948, TK2510. $\mathrm{ar}^{+}$Cocos nucifera, AM2955, TK2518. LOC. 49: gl ${ }^{+}$Calophyllum inophyllum, AM2714, TK2234. $\mathrm{w}^{+}$Cocos nucifera, AM2715, TK2236.

P. superbum Hagelst. (Granitic islands: 4 MC, 1 cp. R) LOC. 3: b* SLS26718 (pH 7.6), SLS26739 (pH 7.1), SLS26750 (pH 8.4), SLS26881 (pH 8.3).

P. tenerum Rex (Granitic islands: 4 FC, 2 cp, 2 ps. O) Aldabra atoll 1, 8: $\mathrm{gl}^{+} \operatorname{Cocos} \mathrm{sp} ., 3$ gatherings (Ing \& Hnatiuk 1981). LOC. 36: $\mathrm{w}^{+} \& \mathrm{~b}^{+}$Terminalia catappa, AM3217, TK2780. LOC. 42C: ar* liana on Calophyllum inophyllum, AM2953, TK2516.

P. viride (Bull.) Pers. (Granitic islands: 6 FC, $3 \mathrm{cp}$, 2 ps. C)

Aldabra atoll 1, 4, 8, 13: gl ${ }^{+}$Cocos sp., $\mathrm{w}^{+}$Sideroxylon sp., 19 gatherings (Ing \& Hnatiuk 1981). LOC. 9: $\mathrm{w}^{+}$Swietenia macrophylla, AM3050, TK2800. LOC. 22F: $\mathrm{w}^{+}$palm, AM2736, TK2071. LOC. 21 F: $\mathrm{w}^{+}$Terminalia catappa, AM2766, TK2180.

P. viride var. aurantium (Bull.) Lister (Granitic islands: $26 \mathrm{FC}, 11 \mathrm{cp}, 9 \mathrm{ps}$. C)

LOC. 9: $\mathrm{w}^{+}$Bambousa sp., AM3054, TK2806. LOC. 8: b* Nephrosperma vanhoutteanum, AM3096, TK2909. $\mathrm{w}^{+}$Spondias cytherea, AM3102, TK2916. LOC. 25A: ar $^{+}$Cocos nucifera, AM2755, TK2130. LOC. 27B: $\mathrm{w}^{+}$Artocarpus altilis, AM3169, TK2642. LOC. 37B: $\mathrm{w}^{+} \& \mathrm{~b}^{+}$Calophyllum inophyllum, AM3192, TK2690. $\mathrm{w}^{+}$Cinnamomum verum, AM3180, TK2688. LOC. 37E: $\mathrm{w}^{+}$Falcataria moluccana, AM2993, TK2573. LOC. 38A: $\mathrm{w}^{+}$Cinnamomum verum, AM2905, TK2445. LOC. 39B: $\mathrm{w}^{+}$Calophyllum inophyllum, AM2984, TK2556. LOC. 40C: $\mathrm{w}^{+}$Cinnamomum verum, AM2922, TK2468. LOC. 42C: $\mathrm{w}^{+}$Terminalia catappa, AM2944, TK2504. $\mathrm{w}^{+}$Terminalia catappa, AM2963, TK2527.
Reticularia olivacea (Ehrenb.) Fr. (Granitic islands: $1 \mathrm{MC}, 1 \mathrm{cp}, 1$ ps. S)

LOC. 22B: b* Calophyllum inophyllum, SLS32452 (pH 5.7).

Stemonaria longa (Peck) Nann.-Bremek., R. Sharma \& Y. Yamam. (Granitic islands: 6 FC, 2 cp, 2 ps. O)

LOC. 28B: $\mathrm{w}^{+}$Terminalia catappa, AM2929, TK2476. LOC. 43: $\mathrm{w}^{+} \& \mathrm{~b}^{+}$Thespesia populnea, AM2939, TK2496. $\mathrm{w}^{+} \& \mathrm{~b}^{+}$, AM3154, TK2585.

Stemonitis axifera (Bull.) T.Macbr. (Granitic islands: $14 \mathrm{FC}, 6 \mathrm{cp}, 3$ ps. O)

LOC. 9: $\mathrm{w}^{+}$Swietenia macrophylla, AM3046, TK2794. LOC. 6F: $\mathrm{w}^{+}$, AM3071, TK2836. LOC. 22F: $\mathrm{w}^{+}$Cinnamomum verum, AM2734, TK2069. LOC. 37M: $\mathrm{w}^{+}$, AM2877, TK2404. LOC. 38A: $\mathrm{w}^{+}$Cinnamomum verum, AM2910, TK2447. LOC. 40C: $\mathrm{w}^{+} \& \mathrm{~b}^{+}$ Calophyllum inophyllum, AM2919, AM2928, TK2463, TK2474.

S. flavogenita E. Jahn (Granitic islands: 2 FC, 1 cp., R) Aldabra atoll 1: $\mathrm{gl}^{+}$Cocos sp. \& Ochna sp., 2 gatherings (Ing \& Hnatiuk 1981). LOC. 37M: $\mathrm{W}^{+}, \mathrm{b}^{+}$, $\mathrm{gl}^{+}, \mathrm{AM} 2876$, TK2402.

S. fusca Roth (Granitic islands: $18 \mathrm{FC}, 9 \mathrm{cp}, 3$ ps. C)

Aldabra atoll 4, 10: $\mathrm{w}^{+}$Apodytes sp., 2 gatherings (Ing \& Hnatiuk 1981). LOC. 3: $\mathrm{W}^{+}$, AM2182, TK96S. LOC. 5: $\mathrm{w}^{+}, \mathrm{AM} 2107$, TK21S. LOC. 20: $\operatorname{moss}$ on $\mathrm{w}^{+} \&$ $\mathrm{b}^{+}$Calophyllum inophyllum, AM2776, TK2191. LOC. 21 B: $\mathrm{w}^{+}$Terminalia catappa, AM2791, TK2217. LOC. 27B: $\mathrm{w}^{+}$Calophyllum inophyllum, AM3170, TK2644. LOC. 36: $\mathrm{w}^{+}$Terminalia catappa, AM3216, TK2784. LOC. 37N: moss on $\mathrm{w}^{+} \& \mathrm{~b}^{+}$Falcataria moluccana, AM2988, TK2577. LOC. 40C: $\mathrm{w}^{+}$Calophyllum inophyllum, AM2920, TK2464. LOC. 43: $\mathrm{w}^{*} \& \mathrm{~b}^{*}$ Calophyllum inophyllum, AM2937, TK2507.

S. fusca var. nigrescens (Rex) Torrend (Granitic islands: $10 \mathrm{MC}, 6 \mathrm{cp}, 6$ ps. O)

LOC. 6D: ar $^{+}$Dioscorea sp., SLS32379 (pH 6.7). LOC. 22C: $\mathrm{gl}^{+}$Adenanthera pavonina, SLS32381 (pH 5.2). LOC. 22D: gl ${ }^{+}$, SLS32494 (pH 5.5). LOC. 23: $\mathrm{gl}^{+}$Lodoicea maldivica, SLS32490 ( $\mathrm{pH} 5.5$ ), SLS32494 (pH 5.4). LOC. 37C: $\mathrm{ar}^{+}$Cinnamomum verum, SLS32333 ( $\mathrm{pH} 4.6)$, SLS32338 (pH 4.6). b* Cinnamomum verum, SLS32377 (pH 5.2). LOC. 47A: 
ar $^{+}$Phoenicophorium borsigianum, SLS32331 (pH 4.9), SLS32345 (pH 4.7).

S. herbatica Peck (Granitic islands: 1 MC, 1 cp, 1 ps. R) Aldabra atoll 1: gl ${ }^{+}$Tournefortia argentea, 2 gatherings (Ing \& Hnatiuk 1981). LOC. 2: ar $^{+}$Epipremnum pinnatum, SLS32383 ( $\mathrm{pH}$ 5.5).

S. pallida Wingate (Granitic islands: $6 \mathrm{FC}, 3 \mathrm{cp}$, 2 ps. O)

LOC. 22F: $\mathrm{w}^{+}$palm, AM2737, TK2072. LOC. 22G: $\mathrm{w}^{+}$Calophyllum inophyllum, AM2727-1, TK2061. LOC. 40A: $\mathrm{w}^{+} \& \mathrm{~b}^{+}$Terminalia catappa, AM2914, TK2452.

S. pallida var. rubescens Y. Yamam. (Granitic islands: $2 \mathrm{FC}, 1 \mathrm{cp}, 1 \mathrm{ps} . \mathrm{R}$ )

LOC. 20: $\mathrm{w}^{+}$Calophyllum inophyllum, AM2787, TK2206.

S. splendens Rostaf. (Granitic islands: 28 FC, $12 \mathrm{cp}$, 5 ps. C)

Aldabra atoll 1: $\mathrm{w}^{+}$Casuarina sp., 1 gathering (Ing \& Hnatiuk 1981). LOC. 2: $\mathrm{w}^{+}$Tabebuia pallida, AM3094, TK2889. LOC. 22D: $\mathrm{w}^{+}$Falcataria moluccana, AM2745, TK2081. LOC. 22F: $\mathrm{w}^{+}$Cinnamomum verum, AM2735, TK2070. LOC. 21F: ar ${ }^{+}$Calophyllum inophyllum, AM2772, TK2187. LOC. 31C: $\mathbf{w}^{+}$, AM2780, TK2292. LOC. $32: \mathrm{w}^{+} \& \mathrm{~b}^{+}$Terminalia catappa, AM2916, TK2455. LOC. 34A: $\mathrm{w}^{+}$Terminalia catappa, AM2844, TK2353. LOC. 37B: $\mathrm{w}^{+} \& \mathrm{~b}^{+}$Calophyllum inophyllum, AM3187, TK2694. LOC. 37-l: $\mathrm{w}^{+}$ \& $\mathrm{b}^{+}$Cinnamomum verum, AM3176, TK2678. LOC. 37G: b ${ }^{+}$Falcataria moluccana, AM2861, TK2379. $\mathrm{w}^{+}$Falcataria moluccana, AM2992, TK2571. LOC. 37M: b ${ }^{+}$, AM2876, TK2403. $\mathrm{b}^{+}$Falcataria moluccana, AM2858, TK2372. LOC. 49: $\mathrm{b}^{+}$Calophyllum inophyllum, AM2712, TK2233.

Stemonitopsis gracilis (G. Lister) Nann.-Bremek. (Granitic islands: $2 \mathrm{FC}, 1 \mathrm{cp}, 1 \mathrm{ps}$. R)

LOC. 42B: $\mathrm{w}^{+}$Calophyllum inophyllum, AM2946, TK2509.

Stemonitopsis hyperopta (Meyl.) Nann.-Bremek. (Granitic islands: 4 FC, 2 cp, 1 ps. R)

LOC. 39C: $\mathrm{w}^{+}$Calophyllum inophyllum, AM2973, TK2544. LOC. 41B: $\mathrm{w}^{+}$Calophyllum inophyllum, AM2971, TK2541.
S. typhina (F.H. Wigg.) Nann.-Bremek. (R)

Aldabra atoll 1, 4, 8: $\mathrm{w}^{+}, \mathrm{gl}^{+}$Cocos $\mathrm{sp} ., 4$ gatherings (Ing \& Hnatiuk 1981).

Trichia decipiens (Pers.) T. Macbr. (Granitic islands: $1 \mathrm{MC}, 1 \mathrm{cp}, 1$ ps. S)

LOC. 23: $\mathrm{b}^{+}$Tabebuia pallida, SLS32449 (pH 5.6).

Tubifera ferruginosa (Batsch) J.F. Gmel. (Granitic islands: 4 FC, $2 \mathrm{cp}, 1$ ps. R)

LOC. 3: b*, AM2179, TK93S. LOC. 24: $\mathrm{w}^{+}$ Tabebuia pallida, AM2761, TK2168.

T. microsperma (Berk. \& M.A. Curtis) G.W. Martin (Granitic islands: $5 \mathrm{FC}, 3 \mathrm{cp}, 2 \mathrm{ps}$. R)

LOC. 6B: $\mathrm{w}^{+}$, AM 3120. LOC. 33: $\mathrm{w}^{+}$, AM2906, TK3814. LOC. 37B: $\mathrm{w}^{+}$Calophyllum inophyllum, AM3182, TK3172.

\section{Discussion}

In general, this annotated checklist clearly shows that isolated tropical islands can support a diverse assemblage of myxomycetes. From the total of 143 taxa, 88 species and infra-specific taxa are new for the Seychelles as result of expeditions to Mahé, Praslin, La Digue, Curieuse and Félicité. Fifty-five species had been reported previously from the coralline Aldabra atoll (Ing \& Hnatiuk 1981). Collectively, these records represent six orders, 12 families and 29 genera in the class Myxomycetes, with a predominance of members of the order Physarales (74 taxa). From the total body of data, $4 \%$ species were abundant, $12 \%$ species were common, $29 \%$ were found occasionally, $42 \%$ were rare, and $13 \%$ species had only a single record. The most abundant species were Arcyria cinerea, A. denudata, Diderma effusum, Hemitrichia calyculata, Physarum compressum, and $P$. melleum. A consideration of the taxonomic structure of the myxomycete biota in the area studied shows that the main genera are Physarum (38 species and two varieties), Didymium (17 species), Cribraria (11 species), Arcyria (eight species) and Stemonitis (six species and two varieties) (Table 1). 

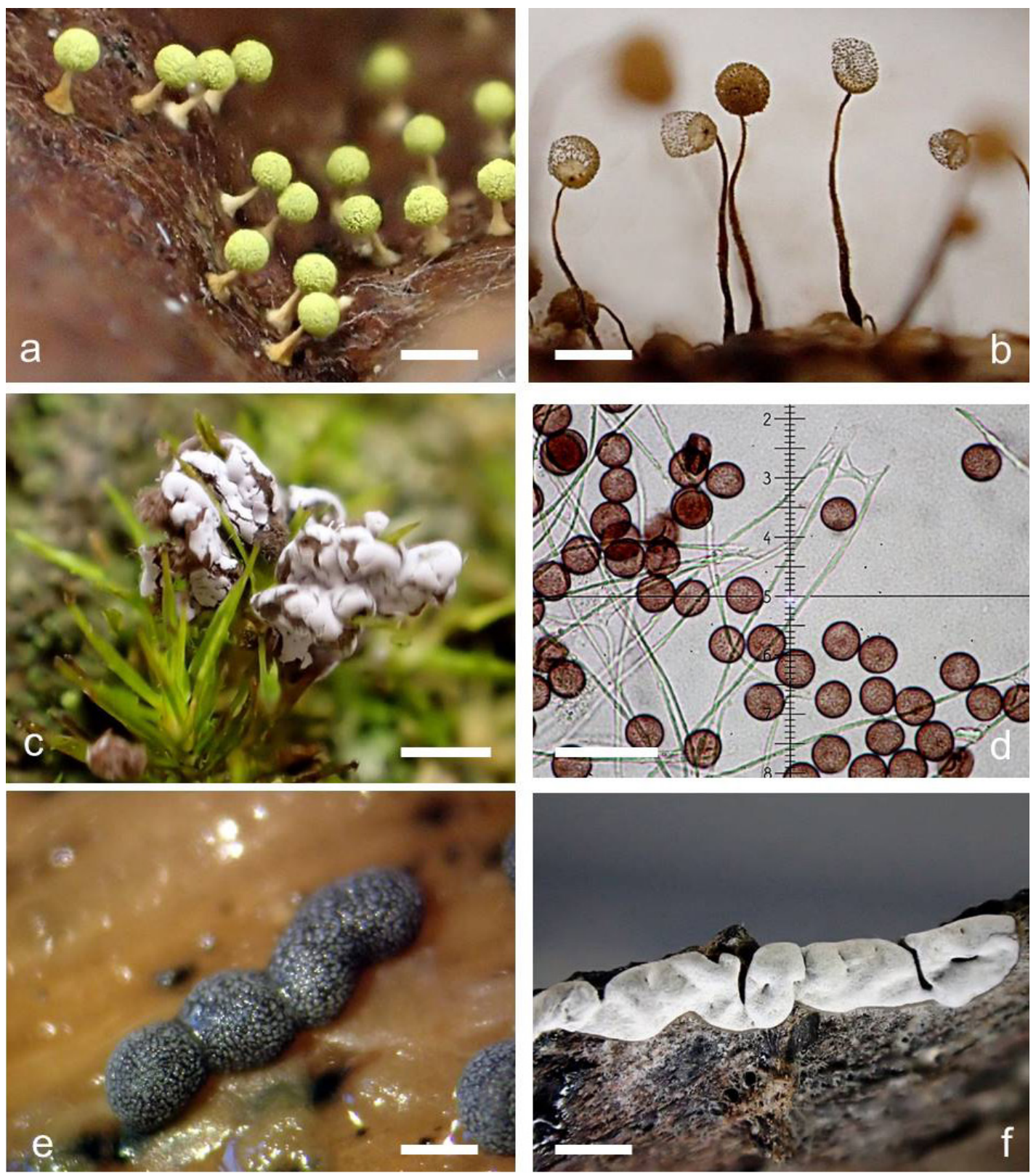

Fig. 2. Myxomycete species collected in the Seychelles. a: Physarum cremiluteum on dead leaves of Terminalia catappa, La Digue, 06-I-2016 (AM2900), b: Cribraria aurantiaca on the trunk of Falcataria moluccana, La Digue, 03-I-2016 (AM2860), c: Diderma saundersii on mosses on Cinnamomum verum, $\mathbf{d}$ : Diderma saundersii capillitium and spores (x630), Mahé, 26-VI-2016 (AM3075), e: Physarum cinereum on dry twigs of Epipremnum pinnatum, La Digue, MC (AM 3899), f: Diderma chondrioderma on the bark of living Rhizophora mucronata, Curieuse, MC (AM2834). Scale bars: a scale $=1 \mathrm{~mm}, \mathrm{~b}$ scale $=0.5 \mathrm{~mm}$. c scale $=0.5 \mathrm{~mm}, \mathrm{~d}$ scale $=25 \mu \mathrm{m}$, e scale $=0.8 \mathrm{~mm}, f$ scale $=1 \mathrm{~mm}$. - Photo: A. Michaud. 
For six islands (Aldabra, Mahé, Praslin, La Digue, Curieuse and Félicité) only a single species of myxomycete (Physarum crateriforme) was shared in common. For La Dique, Mahé, Praslin and Aldabra, 18 species of myxomycetes were recorded for all of the islands. These were Arcyria cinerea, A. denudata, Ceratiomyxa fruticulosa, Cribraria microcarpa, C. violacea, Diderma effusum, Didymium squamulosum, Hemitrichia calyculata, H. serpula, Lycogala epidendrum, Perichaena depressa, Physarum album, P. compressum, P. crateriforme, P. melleum, P. pusillum, Stemonitis fusca, S. splendens. In total, 50 different localities with 90 collecting plots were subjected to some sampling on the granitic islands. The abundance of myxomycetes collected in the field depends greatly upon the humidity of the collecting locality and the substrate moisture at the time of sampling. Thirty-two percent of all samples were collected in coastal vegetation, $30 \%$ in lowland localities, $19 \%$ at intermediate forests, $9 \%$ in riverine forests, $8 \%$ in mountain forests, and only $2 \%$ in mangrove swamps. The overwhelming majority of species of myxomycetes were found in coastal and lowland vegetation, Cribraria pachydictyon, Didymium clavus, D. minus, D. ovoideum, Physarum atroviolaceum, and P. plicatum were recorded only at high elevations on Mahé. Among the species peculiar for intermediate forests, Arcyria marginoundulata, Diderma rimosum, Comatricha laxa, and Cribraria confusa were noteworthy examples.

Reach vegetation in the Seychelles is characterized by many endemic and introduced plants and thus substrates diversity for myxomycetes is high (Fig. 3). However, from 57 species of plants used by myxomycetes as substrates on the granitic islands, only nine species provided $63 \%$ of the specimens of myxomycetes. The most productive substrates on the islands were provided by the common Seychelles trees Calophyllum inophyllum, Cinnamomum verum, Cocos nucifera, Falcataria moluccana, Synogonium podophyllum, Tabebuia pallida, Terminalia catappa, and Thespesia populnea. It is noteworthy that among singleton substrates, there is no single example specific for any myxomycetes recorded only once, and most single and rare species usually were found on substrates that were abundant and common. Almost $30 \%$ of the specimens of myxomycetes were collected on dead wood and large decaying palm stems, nearly equal numbers of species were found on bark and ground litter $(26 \%$ and $24 \%$, respectively), and $20 \%$ species were associated with aerial substrates. Some species were not very selective with respect their choice of substrates, but others clearly preferred certain substrates.

In summary, because of favorable climatic conditions and their location between Asia and Africa, the Seychelles Islands are very promising for the study of myxomycetes. There is still the hope that the vegetation of the Seychelles could yield interesting results for species of myxomycetes and thus there is a need for more detail research. Future expeditions should be carried out to survey islands not yet explored and high elevations areas that were underrepresented in the expeditions reported herein. 

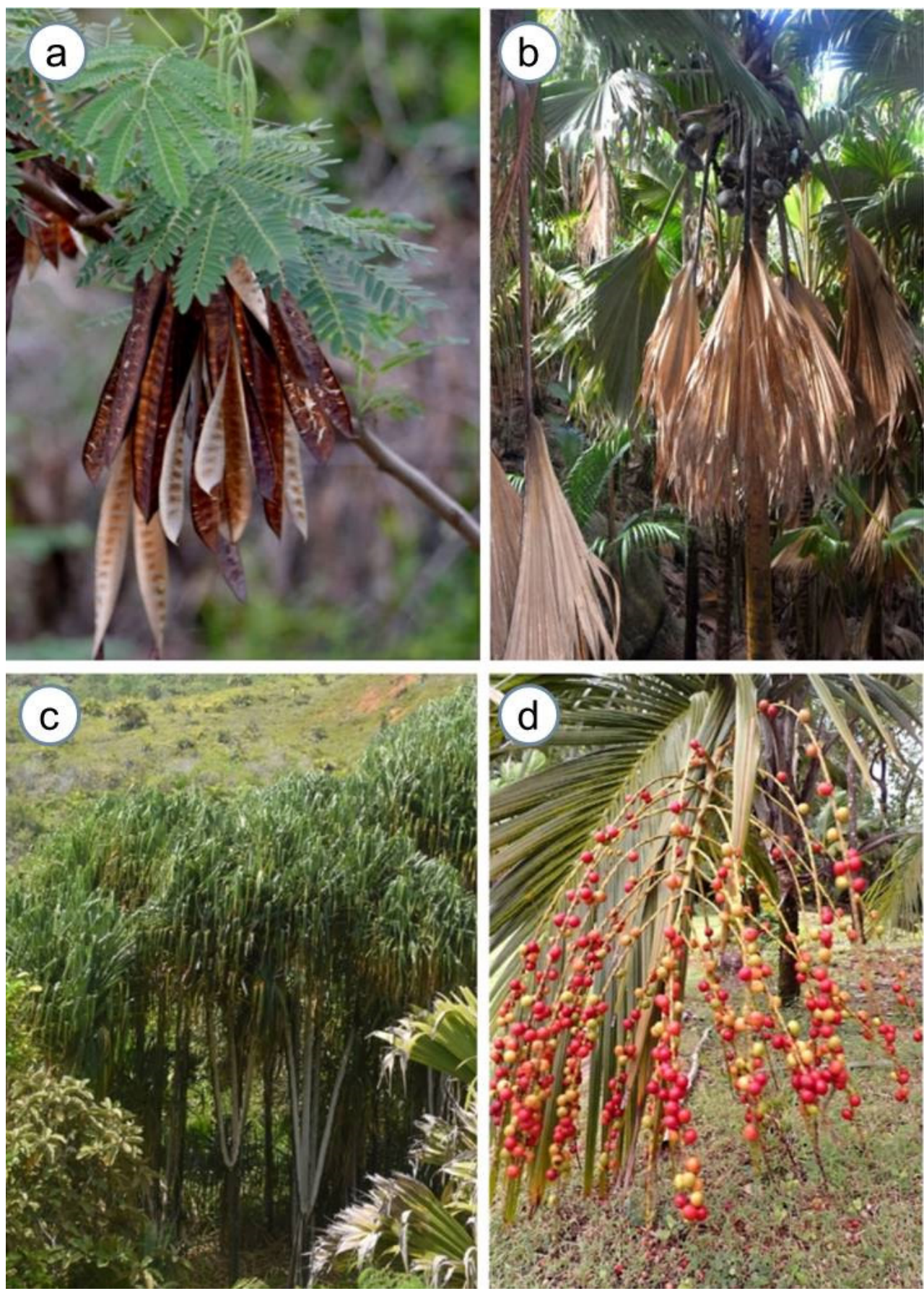

Fig. 3. Examples of substrates for myxomycetes. $\boldsymbol{a}$ : Leucaena leucocephala, Praslin, $\boldsymbol{b}$ : Lodoicea maldivica (endemic) in Vallée de Mai, a World Heritage Site, Praslin, c: Martellidendron hornei (endemic), La Plaine hollandaise, Praslin, d: inflorescence with ripe fruits of Nephrosperma vanhoutteanum (endemic), Mahé, National Biodiversity Centre. Photo: A. Michaud. 
Table 1. Taxonomical structure of an annotated checklist of myxomycetes from the Seychelles Islands

\begin{tabular}{|c|c|c|c|}
\hline Order & Family & Genus & Number of taxa \\
\hline Ceratiomyxales & Ceratiomyxaceae & Ceratiomyxa & 2 \\
\hline \multirow[t]{6}{*}{ Cribrariales } & Cribrariaceae & Cribraria & 11 \\
\hline & Dictydiaethaliaceae & Dictydiaethalium & 1 \\
\hline & Liceaceae & Licea & 6 \\
\hline & Reticulariaceae & Lycogala & 2 \\
\hline & & Reticularia & 1 \\
\hline & & Tubifera & 2 \\
\hline \multirow[t]{2}{*}{ Echinosteliales } & Clastodermataceae & Clastoderma & 1 \\
\hline & Echinosteliaceae & Echinostelium & 2 \\
\hline \multirow[t]{8}{*}{ Physarales } & Didymiaceae & Diachea & 3 \\
\hline & & Diderma & 5 \\
\hline & & Didymium & 17 \\
\hline & Physaraceae & Badhamia & 1 \\
\hline & & Craterium & 3 \\
\hline & & Fuligo & 4 \\
\hline & & Physarella & 1 \\
\hline & & Physarum & 40 \\
\hline \multirow[t]{7}{*}{ Stemonitidales } & Stemonitidaceae & Collaria & 1 \\
\hline & & Comatricha & 5 \\
\hline & & Lamproderma & 1 \\
\hline & & Macbrideola & 2 \\
\hline & & Stemonaria & 1 \\
\hline & & Stemonitis & 8 \\
\hline & & Stemonitopsis & 3 \\
\hline \multirow[t]{5}{*}{ Trichiales } & Arcyriaceae & Arcyria & 8 \\
\hline & & Perichaena & 7 \\
\hline & Trichiaceae & Hemitrichia & 3 \\
\hline & & Metatrichia & 1 \\
\hline & & Trichia & 1 \\
\hline
\end{tabular}




\section{Acknowledgements}

The authors are very thankful to Marianne Meyer for her highly valuable comments relating to the identification of the most difficult specimens of myxomycetes. Thanks also to all our colleagues who were interested in and commented this research in the Seychelles.

\section{References}

Adamonyte, G., Stephenson, S.L., Michaud, A., Seraoui, E-H., Meyer, M., Novozhilov, Y. K. \& Krivomaz, T. 2011: Myxomycete species diversity on the island of La Réunion

(Indian Ocean). Nova Hedwigia 92: 523-549.

Fleischmann, K., Héritier, P., Meuwly, C., Küffer, C. \& Edwards, P.J. 2003: Virtual gallery of the vegetation and flora of the Seychelles. Bulletin of the Geobotanical Institute ETH 69: 57-64.

Franda, M. 2019: The Seychelles: Unquiet Islands. - New York, Routledge. 140 pp.

Hansen, S.G. \& Laboudallon, V.F. 2013: Flora of the Seychelles. A Field Guide to Selected Plants. - Trykkeriet Friheden Aps, Denmark. 663 pp.

Hill, M.J. 2002: Biodiversity Surveys and Conservation Potential of Inner Seychelles Islands. - National Museum of Natural History, Smithsonian Institution, Washington. 272 pp.

Ing, B. \& Hnatiuk, R.J. 1981: Myxomycetes of Aldabra Atoll. Atoll Research Bulletin 249: 1-10.

Kryvomaz, T., Michaud, A. \& Stephenson, S.L. 2017: First survey of Myxomycetes on Mahé Island in the Seychelles. Nova Hedwigia 104: 65-84.

Kryvomaz, T., Michaud, A. \& Stephenson, S.L. 2020:

Myxomycete biodiversity on five islands of the Seychelles.

Zootaxa 4851: 201-244.

Lado, C. 2005-2020: An on line nomenclatural information system of Eumycetozoa. Real Jardín Botánico, CSIC. Madrid, Spain. https://eumycetozoa.com (Date of access: 09.08.2020).
Macabago, S.A.B., dela Cruz, T.E.E., Stephenson, S.L. 2012: First records of myxomycetes from Lubang Island, Occidental Mindoro, Philippines. Sydowia 64: 109-118.

Macabago, S.A.B., Stephenson, S.L., dela Cruz, T.E.E. 2016: Diversity and distribution of myxomycetes in coastal and mountain forests of Lubang Island, Occidental Mindoro, Philippines. Mycosphere 7: 18-29.

Macabago, S.A.B., Dagamac, N.H.A., dela Cruz, T.E.E., Stephenson, S.L. 2017: Implications of the role of dispersal on the occurrence of litter-inhabiting myxomycetes in different vegetation types after a disturbance: a case study in Bohol Islands, Philippines. Nova Hedwigia 104: 221-236.

Merlin, A., Senterre, B. \& Francisco, E. 2012: Seychelles Plant Gallery. National History Museum, Victoria, Seychelles. Available from http://www.seychellesplantgallery.com (Date of access: 09.08.2020)

Poulain, M., Meyer, M. \& Bozonnet, J. 2011: Les Myxomycètes 2 vols. - Delémont, Sarl Editions FMDS. 1119 pp.

Stephenson, S.L. \& Rojas, C. 2017: Myxomycetes Biology, Systematics, Biogeography and Ecology 1st Edition. - London, Academic Press. 474 pp.

Stephenson, S. L. \& Stephenson, B.C. 2019: Distribution and ecology of myxomycetes on Christmas Island, Indian Ocean. Phytotaxa 416: 138-148.

Stephenson, S. L. \& Stephenson, B.C. 2020: Biodiversity of myxomycetes on Norfolk Island, South Pacific Ocean. Cunninghamia 20: 115-121.

Stephenson, S.L., Kalyanasundaram, I. \& Lakhanpal, T. N. 1993: A Comparative biogeographical study of Myxomycetes in the mid-Appalachians of eastern North America and two regions of India. Journal of Biogeography 20: 645.

Stoddart, D.R. 1984: Biogeography and Ecology of the Seychelles Islands. - Monographiae Biologicae, Boston \& Lancaster. 691 pp. 